Bull. Austral. Math. Soc.

Vol. 41 (1990) [1-44]

\title{
APPROXIMATE METHODS FOR MODELLING CAVITATION BUBBLES NEAR BOUNDARIES
}

\author{
A. KuCERA AND J.R. Blake
}

\begin{abstract}
Approximate methods are developed for modelling the growth and collapse of clouds of cavitation bubbles near an infinite and semi-infinite rigid boundary, a cylinder, between two flat plates and in corners and near edges formed by planar boundaries. Where appropriate, comparisons are made between this approximate method and the more accurate boundary integral methods used in earlier calculations. It is found that the influence of nearby bubbles can be more important than the presence of boundaries. In confined geometries, such as a cylinder, or a cloud of bubbles, the effect of the volume change due to growth or collapse of the bubble can be important at much larger distances. The method provides valuable insight into bubble cloud phenomena.
\end{abstract}

\section{INTRODUCTION}

The possibility of the existence of cavitation-like phenomena was postulated by the great Euler in a memoir on hydraulics in 1754, while they were first described by the fluid dynamicist Osborne Reynolds in 1894 when he observed bubbles forming in water flowing through a constriction in a tube. Several years after Reynold's paper was published, a very strange destruction of the propellers on fast steamboats was observed, examples being the ocean liners Lusitania and Mauritania. In some cases, a few hours running time was sufficient to make the propellers unfit for operation. In 1915 the British Admiralty instituted a special commission to determine the mechanisms causing the destruction of ship propellers. They reported that the damage was due to hydraulic blows accompanying the collapse of the cavities. Similar phenomena occurred in turbomachinery. Not only are bubbles capable of causing damage but they may also diminish the performance of the hydraulic device.

Theoretical understanding of cavitation is often associated with the cavitation number $K$,

$$
K=\frac{p_{0}-p_{c}}{(1 / 2) \rho U^{2}}
$$

Received 9 December 1988.

This work was supported by the Australian Research Grants Committee

Copyright Clearance Centre, Inc. Serial-fee code: 0004-9729/90 \$A2.00+0.00. 
where $p_{0}$ is the static pressure; $p_{c}$, the saturated vapour pressure inside the bubble; $\rho$, the density of the liquid and $U$, the incident velocity. Experimentally cavitation is observed to occur at a critical value of $K_{c}$ (for example $K_{c}=0.33$ for a 15 caliber ogive, Plesset [12]). Three regimes of flow are observed to exist: for $K>K_{c}$, no cavitation occurs; for a range of $K$ marginally less than $K_{c}$, bubbles are observed while for smaller values of $K$, a large "steady" cavity is observed. In our study of cavitation we are primarily concerned with the intermediate stage when bubbles are formed. This type of flow is typical for flow around impeller blades and propellers and over other high speed underwater devices such as torpedoes. Thus, in this case, interest is centred on the growth and collapse of bubbles near a rigid boundary due to a varying pressure field. Inception and growth occurs when the dynamic pressure falls below the vapour pressure for sufficient time, whereas collapse will occur when the pressure rises. For theoretical studies of this type of problem we can model the flow by considering a bubble, which is typically very small ( $<1 \mathrm{~mm}$ ), near either an infinite plane boundary or near a semi-infinite boundary for leading and trailing edge considerations.

Cavitation phenomena can also occur in other geometries as well. For example in nuclei-counting equipment, flow occurs in a venturi-like cylinder (very similar to the original Reynolds [17] experiments on the 'boiling' of water at ordinary temperatures; see also Chahine and Shen [7]). Cavitation in lubricating bearings is a major problem, which may be modelled as flow between two plane boundaries. As well, cavitation can occur near corners and edges. Later in this paper we will consider examples on the growth and collapse of bubbles in a static situation in all these geometries.

Theoretical developments on bubble dynamics have been concentrated on two aspects of the flow. In the first approach the assumption is made that the bubbles do not substantially affect the ambient pressure field. The mathematical models that have developed at this level usually assume that the bubbles remain spherical and that the only interaction occurs through the ambient pressure field (see for example Plesset [12]). In its most general case the theory includes the inertia of the liquid, viscosity and surface tension of the fluid and can also include the thermodynamic properties of the gas and vapour inside the bubble. Much of the practical pump design theory is based on this approach.

The second approach has been to consider the growth and collapse of a cavitation bubble near a boundary. The principal concern here has been gaining an understanding of the important physical mechanisms associated with the collapse of cavitation bubbles immediately adjacent to a boundary. Since the advent of high speed cameras and fast digital computers there has been a significant experimental and theoretical input into this area. A review of much of this work may be found in the paper by Blake and Gibson [4]. More recent developments on the experimental side with regard to 
damage mechanisms has been reported in Tomita and Shima [21], and more recently again, on composite boundaries by Shima, Tomita, Blake and Gibson [19]. The recent theoretical developments have exploited the boundary integral method to model the axisymmetric growth and collapse of bubbles near different boundaries, subject to the fluid surrounding the bubble being given a finite amount of kinetic energy but being restrained by a constant pressure in the far-field (see for example Blake, Taib and Doherty [5]). Alternatively, only the collapse phase of an initially spherical bubble due to a constant far-field pressure is considered (see for example Plesset and Chapman [13], Prosperetti [14]). Of course, in practice, the flow-field is not likely to remain axisymmetric in the presence of a predominantly tangential flow with pressure gradients also acting in this direction. The presence of nearby bubbles will also have an effect on the growth and collapse characteristics of a bubble.

In summary the main theoretical developments to date have either been the simple "source-sink" approach of the Rayleigh-Plesset equations or the relatively sophisticated boundary integral method employed in the axisymmetric studies. For much of the lifetime of a bubble it remains approximately spherical. It would therefore appear that the growth and early collapse of a cavitation bubble may be well approximated by the first few terms in a spherical harmonic expansion about the bubble centroid, but also including any image terms that may be necessary to satisfy the boundary conditions. Physically the first terms corresponds to the "source-sink" growth and collapse behaviour while the second term, the dipole term, corresponds to a translational motion. Thus in our approximate models we model the behaviour of the bubble by locating a source and dipole at the centroid of the bubble, satisfying the boundary conditions in a least squares sense at a large number of points on the surface of the bubble. These calculations are compared with the more accurate boundary integral method where possible from the perspective of both the shape and conservation of the Kelvin impulse.

In the next section we develop the theory briefly while in later sections we apply these ideas to a range of geometries and examples.

\section{EQUations of Motion}

It is assumed that the fluid is incompressible, inviscid and irrotational, and that surface tension and buoyancy forces are negligible for the major part of the bubble's lifetime (for a more detailed discussion see Blake and Gibson [4] and Gibson and Blake [9]). Under these assumptions the velocity $u$ may be represented by

$$
\mathbf{u}=\nabla \phi
$$

where the potential $\phi$ satisfies,

$$
\nabla^{2} \phi=0
$$


The boundary conditions on the bubble surface $S$ are given by,

$$
\begin{cases}\mathbf{U}_{\bullet}(\mathbf{x}) & =\mathbf{u}(\mathbf{x}), \quad \mathrm{x} \in S \\ p & =p_{c}\end{cases}
$$

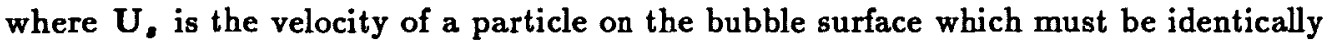
equal to the fluid velocity and where the dynamic pressure $p$ is equal to the saturated vapour pressure $p_{c}$. At "infinity" we require that the velocity tends to zero and the pressure to the static pressure $p_{0}$. For this paper, at least, we assume that the fluid is stationary at infinity, that is

$$
\mathbf{u} \rightarrow 0, \quad p \rightarrow p_{0}
$$

The zero flux boundary conditions on a rigid boundary require that

$$
\frac{\partial \phi}{\partial n}=0, \quad \mathbf{x} \in R
$$

where $R$ is the nearby rigid boundary (see Figure 1 ).

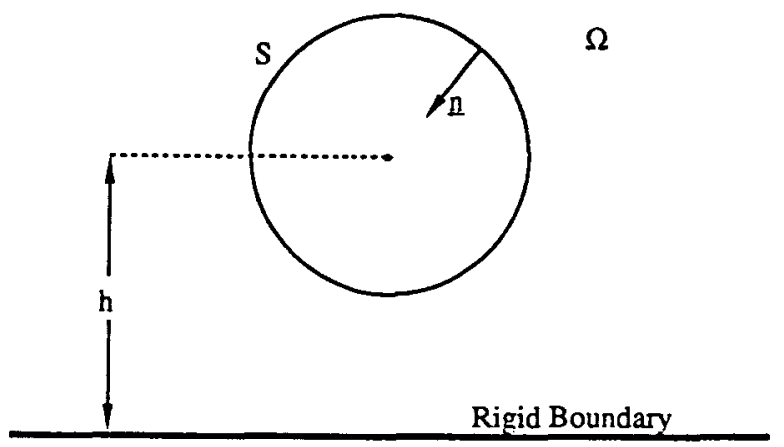

Figure 1 Illustrates the geometry and notation used.

Equating the Bernoulli pressure condition to the vapour pressure on the bubble surface yields,

$$
p_{c}=p_{0}-\rho \frac{\partial \phi}{\partial t}-\frac{1}{2} \rho|\mathbf{u}|^{2}
$$

For the system to be well-posed (that is for a solution to exist for every choice of data and to be unique and continuously dependent on the data), initial conditions must be imposed. We suppose the initial condition is a very small bubble of radius $R_{0}$ on which 
we impose a potential $\phi_{0}$ that can be obtained from the Rayleigh [16] solution for a spherical bubble in an infinite fluid. The potential so obtained is,

$$
\phi_{0}=-R_{0}\left\{\frac{2}{3}\left(\frac{p_{0}-p_{c}}{\rho}\right)\left[\left(\frac{R_{m}}{R_{0}}\right)^{3}-1\right]\right\}^{\frac{1}{2}},
$$

where $R_{m}$ is the maximum bubble radius. The time for a Rayleigh bubble to grow to a radius $R_{0}$ is,

$$
t_{0}=3\left(\frac{3 \rho}{2\left(p_{0}-p_{c}\right)}\right)^{\frac{1}{2}} B_{a}(5 / 6,3 / 2) ; \quad a=\left(\frac{R_{0}}{R_{m}}\right)^{3},
$$

where $B_{a}$ is the incomplete Beta function (Abramowitz and Stegun [1]).

The solution may now formally be specified in terms of the boundary integral representation

$$
c(\mathbf{x}) \phi=\int_{S}\left(\frac{\partial \phi}{\partial n} G-\phi \frac{\partial G}{\partial n}\right) d S,
$$

where $\mathbf{n}$ is the outward normal, $G$ is the appropriate Green's function of Laplace's Equation (2.2) that satisfies the rigid body boundary condition (2.5). The quantity $c(\mathbf{x})$ is defined as follows

$$
c(x)= \begin{cases}1, & x \in \Omega \\ \frac{1}{2}, & x \in S\end{cases}
$$

where $\Omega$ is the domain of the fluid, $S$ the surface of the bubble and $\mathbf{x}$ an arbitrary field point.

If we were considering a bubble in an infinite fluid the Green's function $G$ would be defined by,

$$
G(\mathbf{x}, \mathbf{s})=\frac{1}{4 \pi|\mathbf{x}-\mathbf{s}|}
$$

where $\mathbf{x} \in \Omega$ and $s \in S$. In the far-field, where $|\mathbf{x}|$ is much greater than $|\mathbf{s}|$, the Green's function terms in the integrand of (2.9) may be approximated as follows,

$$
\frac{1}{|x-s|}=\frac{1}{|x|}+\frac{s \cdot x}{|x|^{3}}+0\left(\frac{1}{|x|^{3}}\right) \text {, }
$$

and

$$
\begin{aligned}
\frac{\partial}{\partial n}\left(\frac{1}{|\mathbf{x}-\mathbf{s}|}\right) & =\mathbf{n} \cdot \nabla \cdot \frac{1}{|\mathbf{x}-\mathbf{s}|} \\
& =\frac{\mathbf{n} \cdot \mathbf{x}}{|\mathbf{x}|^{3}}+o\left(\frac{1}{|\mathbf{x}|^{3}}\right),
\end{aligned}
$$


Thus, in the far-field, the potential behaves like,

$$
\phi \sim-\frac{1}{4 \pi}\left\{\frac{m(t)}{|\mathbf{x}|}+\frac{\mathrm{d}(t) \cdot \mathbf{x}}{|\mathbf{x}|^{3}}\right\}+o\left(\frac{1}{|\mathbf{x}|^{3}}\right),
$$

where the source strength is given by

$$
m(t)=-\int_{S} \frac{\partial \phi}{\partial n} d S
$$

and the far-field dipole strength by,

$$
\mathrm{d}(t)=\int_{S}\left(\mathbf{n} \phi-\mathbf{s} \frac{\partial \phi}{\partial n}\right) d S
$$

It may also be shown (see Lighthill [11]) that for an infinite fluid the dipole strength is equal to,

$$
\mathbf{d}(t)=\frac{d}{d t}\left(V_{b} \mathbf{x}_{c}\right)+\frac{1}{\rho} \mathbf{I}
$$

where $V_{b}$ is the volume of the bubble, $\mathbf{x}_{c}$ the position vector of the centroid, and $\mathbf{I}$, the Kelvin impulse which is defined by,

$$
\mathbf{I}=\rho \int_{S} \phi \mathbf{n} d S .
$$

However, these relations are modified for a bounded fluid as discussed in Blake [2].

The approximation (2.14) is also extended into the near-field around the cavitation bubble because for much of its lifetime it is nearly spherical and may be well approximated by the lowest order spherical harmonics. It is also possible to generalise this approach to $N$ bubbles in an infinite fluid where

$$
\phi=\sum_{i=1}^{N} \phi_{i}
$$

with

$$
\phi_{i}=-\frac{1}{4 \pi}\left\{\frac{m_{i}}{\left|\mathbf{x}-\mathbf{x}_{c}^{(i)}\right|}+\frac{\mathrm{d}_{i} \cdot\left(\mathrm{x}-\mathrm{x}_{c}^{(i)}\right)}{\left|\mathbf{x}-\mathbf{x}_{c}^{(i)}\right|^{3}}\right\}
$$

where $m_{i}$ and $d_{i}$ are the source and dipole strength respectively, and $\mathbf{x}_{c}^{(i)}$ is the centroid of the i-th bubble,

$$
\mathbf{x}_{c}^{(i)}=\frac{1}{V_{i}} \int_{D^{(i)}} \mathbf{x d V}, \quad \text { with } \mathbf{x} \in D^{(i)}
$$


In (2.21) $V_{i}$ is the volume, and $D^{(i)}$ the domain of the $\mathrm{i}$-th bubble. The numerical values of $\boldsymbol{m}_{\boldsymbol{i}}$ and $\mathbf{d}_{\boldsymbol{i}}$ are obtained by taking a weighted least squares estimate of the potential over the surfaces of the $N$ bubbles, while the $\mathrm{x}_{c}^{(i)}$ are obtained using a simple numerical integration routine on each bubble. Thus the motion of each bubble can be determined by the scalar source strengths $m_{i}$ and the vector dipole strength $d_{i}$ for every one of the $N$ bubbles. In bounded domains the R.H.S of (2.20) will also include image terms to allow the no flux condition through rigid boundaries to be met. A similar approach to this has been developed by Prosperetti [15] who modelled the growth and collapse of a spheroidal bubble by a finite number of sources, while Blake and Gibson [3] used a line distribution of sources. Shima and Nakajima [18] used a spectral method of 8 terms which also has similarities to this approach.

In developing the numerical solution, it proves convenient to non-dimensionalise the equations and parameters. Length is scaled with respect to $R_{m}$, time with respect to $R_{m} /(\Delta p / \rho)^{1 / 2}$ and pressures with respect to $\left(p_{0}-p_{c}\right)$. In the next sections a range of problems in varying geometries are undertaken.

\section{Method of Solution}

The shape of each bubble is obtained by specifying and following Lagrangian particles on its surface as follows,

$$
\mathbf{X}_{j}^{(i)}=\mathbf{X}\left(\mathbf{s}_{j}^{(i)}\right), \quad i=1, \ldots, N ; \quad j=1, \ldots, M^{(i)},
$$

where the position vector $s_{j}^{(i)}$ of the "particles" is chosen in some ordered manner.

Numerical experimentation has shown that the initial bubble radius $R_{0}$ can reasonably be set at 0.1 which is $10^{-3}$ of the maximum volume. Thus, using Equations (2.7) and (2.8), the nondimensional initial conditions are

$$
R_{0}=0.1, \quad t_{0}=0.0015527, \quad \phi_{0}=-2.5806976,
$$

for all cases. The positions of the centroids are initialised through the input.

The velocity components $\left(u_{j}^{(i)}=\left(u_{x}, u_{y}, u_{z}\right)\right)$ of individual "particles" on the bubble surfaces are calculated by evaluating (2.1) for the appropriate potentials that are specified in the subsequent sections.

With the new velocity components calculated, updating the bubble shapes can easily be done by employing a simple Euler scheme, namely

$$
\mathrm{X}_{j}^{(i)}(t+\Delta t)=\mathrm{X}_{j}^{(i)}(t)+\mathrm{u}_{j}^{(i)} \Delta t+0(\Delta t)^{2},
$$

which is stable for the total lifespan of the bubbles provided $\Delta t$ is chosen correctly. 
To update the potential the substantial derivative must be employed,

$$
\frac{d \phi}{d t}=\frac{\partial \phi}{\partial t}+|\mathbf{u}|^{2}
$$

The partial time derivative term in (3.4) would normally complicate the problem but fortunately it can be eliminated by using the nondimensionalised form of Bernoulli's equation as follows

$$
\frac{\partial \phi}{\partial t}=1-\frac{1}{2}|\mathbf{u}|^{2}
$$

to yield

$$
\frac{d \phi}{d t}=1+\frac{1}{2}|\mathrm{u}|^{2}
$$

Again, employing a simple Euler scheme on Equation (3.4) gives

$$
\phi_{j}^{(i)}(t+\Delta t)=\phi_{j}^{(i)}(t)+\left[1+\frac{1}{2}\left|u_{j}^{(i)}\right|^{2}\right] \Delta t+0(\Delta t)^{2}
$$

which, with $u_{j}^{(i)}$ known, can be used to update the potentials on the bubble surfaces.

In both (3.3) and (3.7) the time step is carefully chosen to control the change in $\Delta \phi$ between the time steps. It has been shown (see Gibson and Blake [3]), that if $\Delta t$ is chosen so that it satisfies the relationship.

$$
\Delta t=\max _{\mathbf{S}} \frac{\Delta \phi}{\left(1+\frac{1}{2}|\mathbf{u}|^{2}\right)},
$$

at each time step, then both (3.2) and (3.7) will remain stable throughout the growth and collapse phases of the bubbles' lifespan. The strength of the sources $m_{i}(t)$, and dipoles $d_{i}(t)$ are found, at each time step, by satisfying the potentials $\phi_{i}$ on the bubble surfaces in a weighted least square sense.

The node points on the bubble surfaces are initially chosen to be equally spaced. However, as the bubbles develop, the nodes tend to migrate and cluster, especially during the collapsing phase, when they drift into the newly formed jets. To compensate for this bias, the least square procedure is weighted according to the distance between the points. With this data on the bubble shape it is a relatively straight forward exercise to provide a graphical representation of sections to provide a 3-D graphics interpretation. 


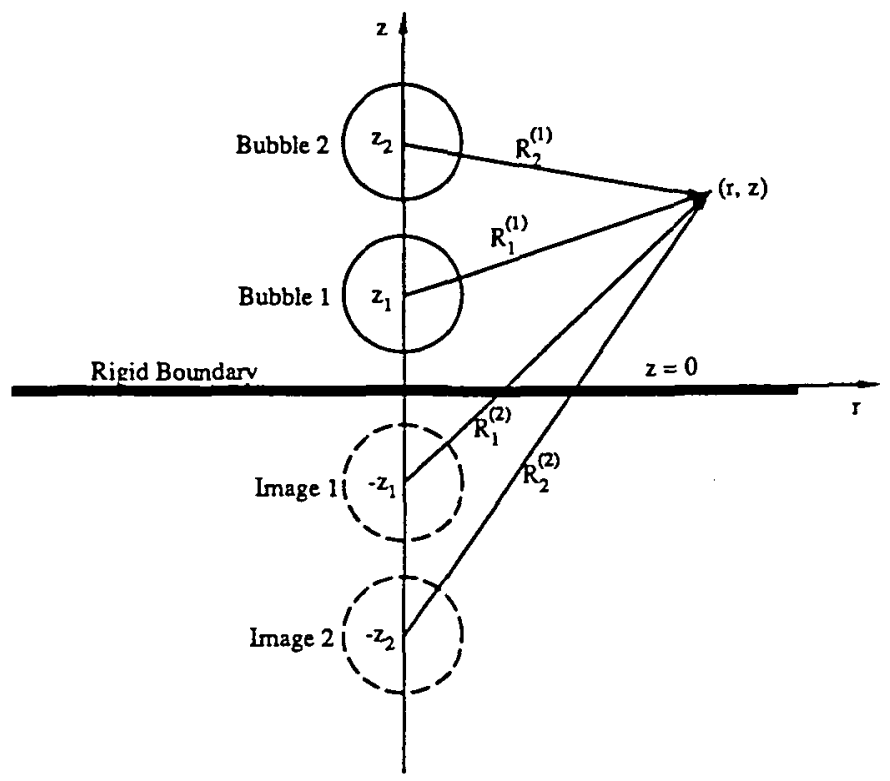

Figure 2 Geometry and image system for two bubbles near an infinite rigid boundary.

\section{AXISYMMETRIC BUBbLES IN A SEMI-INFINITE REgION}

The growth and collapse phase of the axisymmetric bubbles will be modelled using the method of images. Each bubble will be represented by a time dependent source, $m_{i}(t)$, and a time dependent dipole, $d_{i}^{z}(t)$ (for the z-direction motion), and their image in the $\mathrm{z}=0$ plane (see Figure 2).

By superposing the potentials generated by each bubble as specified in (2.19), we have for the case of a semi-infinite region that the individual potential $\phi_{i}$ is given by,

$$
\phi_{i}=-\frac{1}{4 \pi}\left\{m_{i}(t)\left[\frac{1}{R_{i}^{(1)}}+\frac{1}{R_{i}^{(2)}}\right]+d_{i}^{z}(t)\left[\frac{\left(z-z_{i}\right)}{R_{i}^{(1) 3}}-\frac{\left(z+z_{i}\right)}{R_{i}^{(2) 3}}\right]\right\}
$$

with

$$
R_{i}^{(1)}=\sqrt{\left(z-z_{i}\right)^{2}+r^{2}}, \quad R_{i}^{(2)}=\sqrt{\left(z+z_{i}\right)^{2}+r^{2}}
$$

where $z_{i}$ 's are the centroids of the individual bubbles to be evaluated at each time step. Differentiating (4.1) with respect to $r$ and $z$ gives the required individual contri- 
butions to the velocity components as

$$
\begin{aligned}
u_{r}^{(i)}=\frac{\partial \phi_{i}}{\partial r}=\frac{-r}{4 \pi} & \left\{m_{i}(t)\left[\frac{1}{R_{i}^{(1) 3}}+\frac{1}{R_{i}^{(2) 3}}\right]+3 d_{i}^{z}(t)\left[\frac{\left(z-z_{i}\right)}{R_{i}^{(1) 5}}-\frac{\left(z+z_{i}\right)}{R_{i}^{(2) 5}}\right]\right\} \\
u_{z}^{(i)}=\frac{\partial \phi_{i}}{\partial z}=\frac{1}{4 \pi} & \left\{-m_{i}(t)\left[\frac{\left(z-z_{i}\right)}{R_{i}^{(1) 3}}+\frac{\left(z+z_{i}\right)}{R_{i}^{(2) 3}}\right]\right. \\
+ & \left.d_{i}^{z}(t)\left[\left(\frac{1}{R_{i}^{(1) 3}}-\frac{1}{R_{i}^{(2) 3}}\right)-3\left(\frac{\left(z-z_{i}\right)^{2}}{R_{i}^{(1) 5}}-\frac{\left(z+z_{i}\right)^{2}}{R_{i}^{(2) 5}}\right)\right]\right\}
\end{aligned}
$$

whereby the total velocity component of any point is given by

$$
u_{r}=\frac{\partial \phi}{\partial r}=\sum_{i=1}^{N} \frac{\partial \phi_{i}}{\partial r}, \quad u_{z}=\frac{\partial \phi}{\partial z}=\sum_{i=1}^{N} \frac{\partial \phi_{i}}{\partial z}
$$

At each time step the individual centroids $z_{c}^{(i)}$ of the bubbles, are updated for each bubble. With the initial conditions of (3.2) and the specification of the potential in (4.1) and the velocities of (4.3) and (4.4), we are now in a position to model the axisymmetric growth and collapse of a finite number of cavitation bubbles near a rigid boundary by using the approximate method as outlined above.

However, before proceeding to exploit this approach we compare this approximate approach against the more accurate boundary integral method of Blake, Taib and Doherty [5] for a single bubble near a rigid boundary. If the initial location of the centroid is given by $z_{c}=h$, we can define the dimensionless parameter $\gamma$ by,

$$
\gamma=\frac{h}{R_{m}}
$$

In addition to comparing bubble shapes; we can also check on global quantities such as the volume (as related to the source-sink term) and the Kelvin impulse, defined by,

$$
\mathbf{I}=\rho \int_{S} \phi \mathbf{n} d S
$$

Previously we have seen that the Kelvin impulse is related to the dipole strength by the expression, (Blake [3])

$$
d^{z}=\frac{2}{\rho} I_{z}(t)
$$


(a)
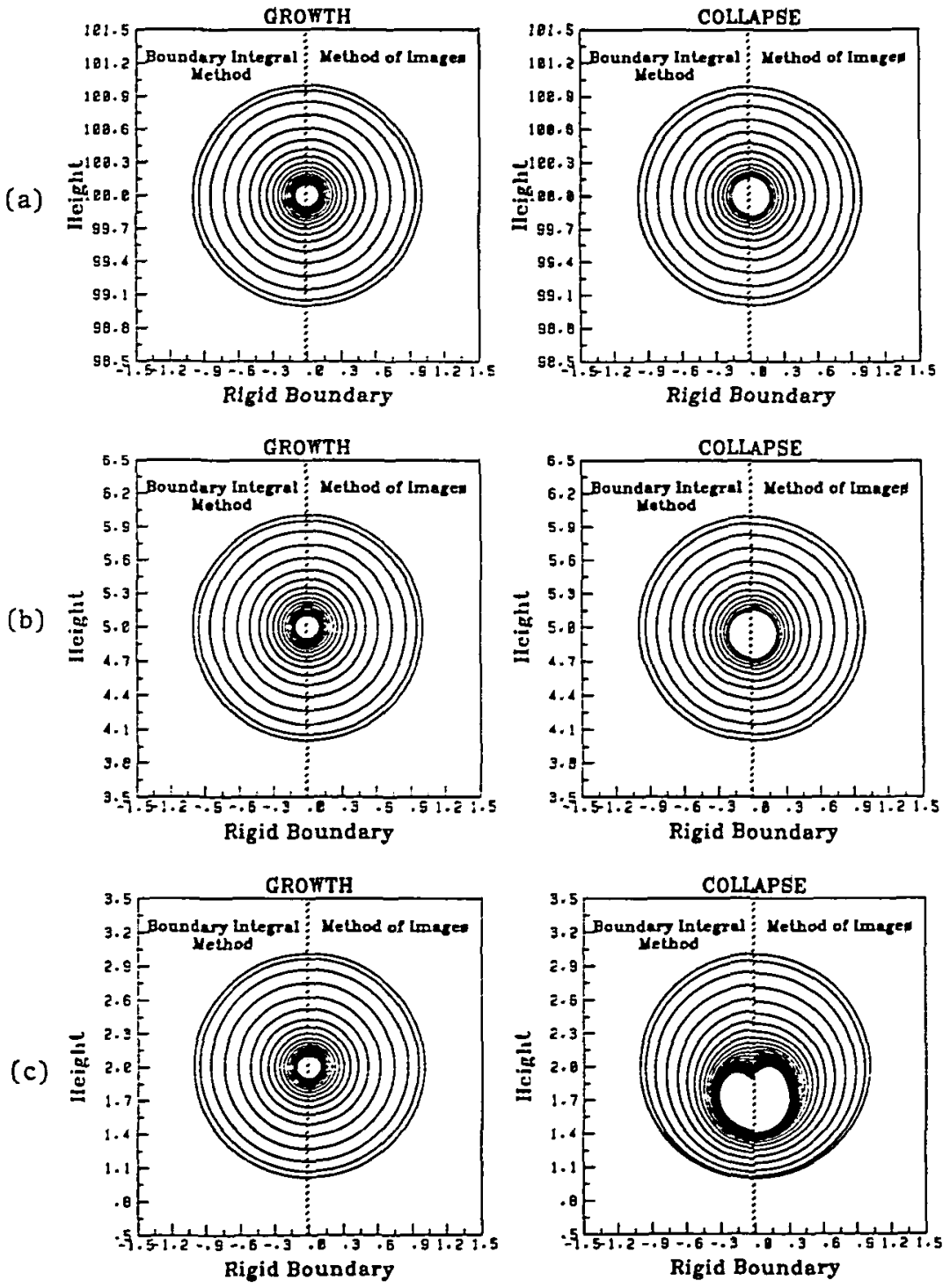

Figure 3.1 Comparisons for the bubble shapes during growth and collapse for $\gamma$ values of 100,5 and 2 .

In Figures 3.1 and 3.2 graphical comparisons are made between the bubble shapes at identical times as well as continuous time comparisons of the bubble volume and Kelvin impulse for initial dimensionless centroid locations of $\gamma=2,5$ and 100 . For $\gamma=100$ we would expect calculations to be close to that of the Rayleigh solution for a 
(a)
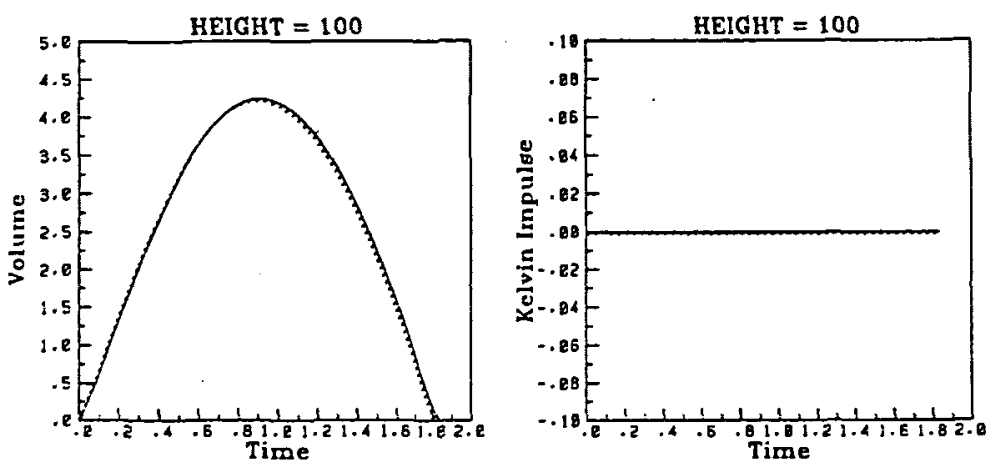

(b)
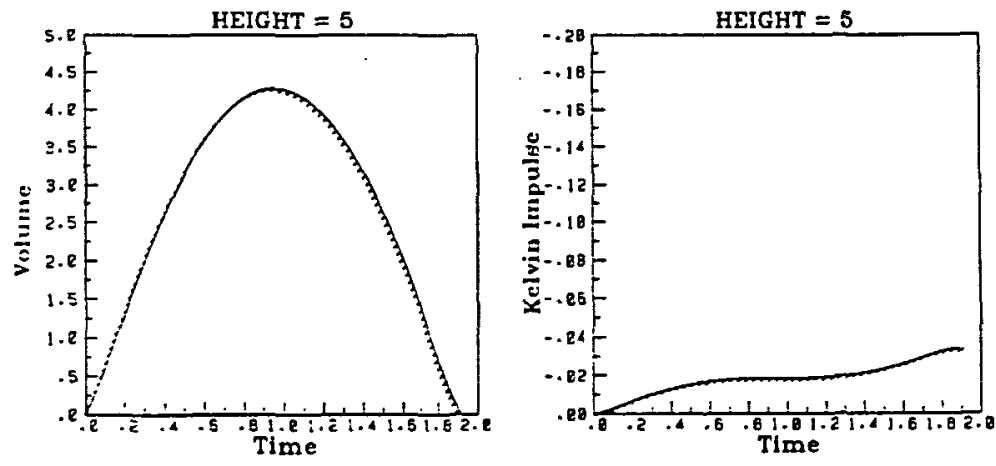

(c)
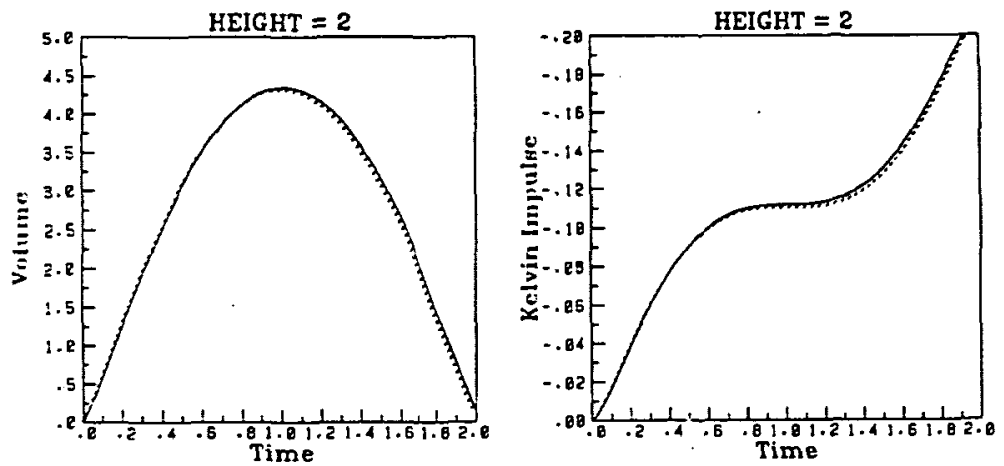

Figure 3.2 Comparisons for the volume and Kelvin impulse for $\gamma$ values of 100,5 and 2. Boundary Integral Method (-), Method of Images (...).

spherical bubble in an infinite fluid. Because the Rayleigh solution is a source-sink flow, we would expect our approximate method to be a very accurate one. Furthermore, the Kelvin impulse in this case should be almost identically zero. It is clear from Figures 
3.1(a) and 3.2(a) that the two methods are extremely close together. At $\gamma=5$ the two approaches also yield similar results. It is only at $\gamma=2$ when the effect of the rigid boundary becomes significant that a variation becomes apparent in the collapse phase. During the expansion phase both results are close together because the bubble remains approximately spherical. However during the collapse phase, a high speed liquid jet is observed to penetrate the bubble in the direction of the rigid boundary. The largest discrepancy appears to be in the bubble shape, although a small variation in the bubble volume and Kelvin impulse is also apparent in Figure 3.2. Numerical comparisons are also provided in Table 1, showing that the error in the Kelvin impulse is at most $3 \%$. It is clear from these comparisons that the approximate method exploiting only the source and dipole term seems to be a remarkably accurate approximation for most of the lifetime of a single bubble provided $\gamma \geqslant 2$.
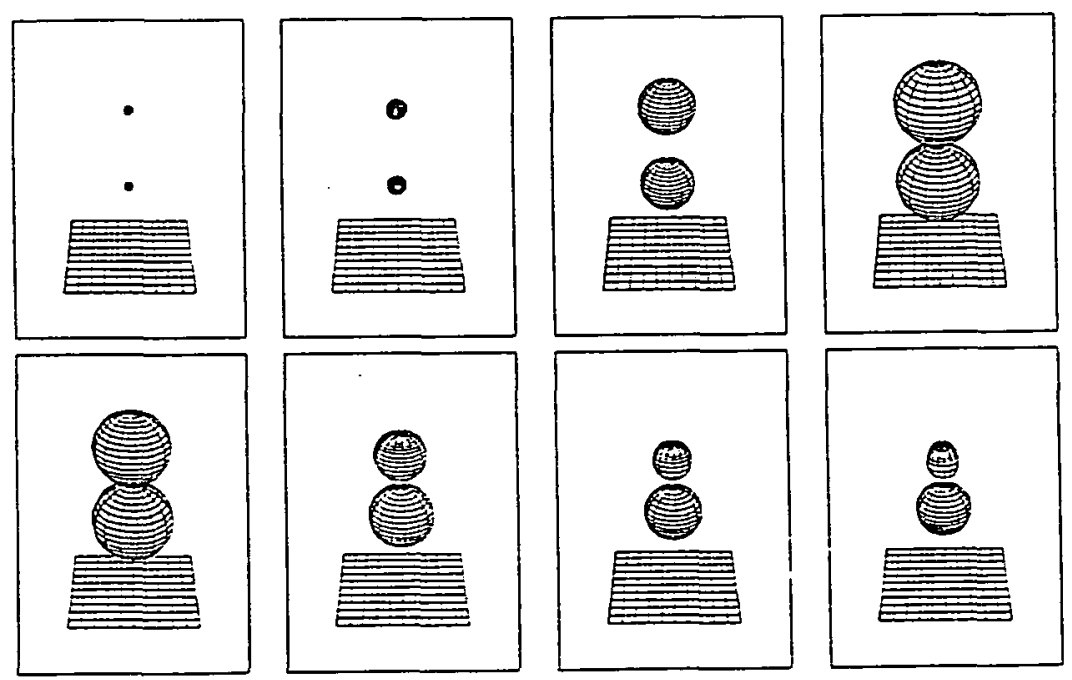

Figure 4.1(a) Three dimensional illustrations of the growth and collapse of two bubbles initially located at $\gamma=2$ and 4 .

In Figure 4.1(a) a 3-D graphical illustration of the growth and collapse of two bubbles, initially located at $\gamma=2$ and 4 , is shown. The bubbles are attracted towards each other as is clearly indicated by the Kelvin impulse graph in $4.2(\mathrm{a})$. The lower bubble has positive values for the Kelvin impulse whereas the uppermost bubble is negative. This clearly illustrates that the bubble $(\gamma=2)$ is much more strongly attracted to the nearby bubble than the rigid boundary. Indeed one may interpret the results from the theory of images that an impermeable plane boundary could equally well be located at $\mathrm{z}=3$, hence the preference for motion in this direction. In Figure 4.1(b), 3 bubbles are considered, located initially at $\gamma=1,3$ and 5 . The two closest bubbles to the boundary 


\begin{tabular}{|c|c|c|c|c|c|c|}
\hline \multirow{2}{*}{$\begin{array}{c}\gamma=100 \\
\text { Time }\end{array}$} & \multicolumn{3}{|c|}{ Volume } & \multicolumn{3}{c|}{ Kelvin lmpulse } \\
\cline { 2 - 6 } & B.I.M. & M.O.I. & Error & B.I.M. & M.O.I. & ETor \\
\hline 0.0017 & 0.0047 & 0.0047 & 0.0000 & 0.0000 & 0.0000 & 0.0000 \\
0.0036 & 0.0166 & 0.0166 & 0.0000 & 0.0000 & 0.0000 & 0.0000 \\
0.0087 & 0.0335 & 0.0335 & 0.0000 & 0.0000 & 0.0000 & 0.0000 \\
0.0258 & 0.1231 & 0.1231 & 0.0000 & 0.0000 & 0.0000 & 0.0000 \\
0.0993 & 0.6021 & 0.6021 & 0.0000 & 0.0000 & 0.0000 & 0.0000 \\
0.4266 & 2.7918 & 2.7934 & 0.0016 & 0.0000 & 0.0000 & 0.0000 \\
1.1250 & 3.9649 & 3.9741 & 0.0091 & 0.0000 & 0.0000 & 0.0000 \\
1.6452 & 1.2535 & 1.2671 & 0.0136 & -0.0001 & -0.0001 & 0.0000 \\
1.7942 & 0.2339 & 0.2451 & 0.0112 & -0.0001 & -0.0001 & 0.0000 \\
\hline
\end{tabular}

\begin{tabular}{|c|c|c|c|c|c|c|}
\hline$\gamma=5$ & \multicolumn{3}{|c|}{ Volume } & \multicolumn{3}{c|}{ Kelvin lmpulse } \\
\cline { 2 - 7 } Time & B.I.M. & M.O.I. & Error & B.I.M. & M.O.I. & Error \\
\hline 0.0017 & 0.0047 & 0.0047 & 0.0000 & 0.0000 & 0.0000 & 0.0000 \\
0.0036 & 0.0116 & 0.0116 & 0.0000 & 0.0000 & 0.0000 & 0.0000 \\
0.0087 & 0.0337 & 0.0337 & 0.0000 & -0.0001 & -0.0001 & 0.0000 \\
0.0262 & 0.1250 & 0.1249 & 0.0000 & -0.0005 & -0.0005 & 0.0000 \\
0.1035 & 0.6224 & 0.6223 & 0.0000 & -0.0029 & -0.0029 & 0.0000 \\
0.4464 & 2.8504 & 2.8517 & 0.0012 & -0.0141 & -0.0142 & 0.0001 \\
1.1488 & 4.0591 & 4.0676 & 0.0085 & -0.0185 & -0.0185 & 0.0000 \\
1.6912 & 1.4316 & 1.4459 & 0.0143 & -0.0288 & -0.0290 & 0.0002 \\
1.8581 & 0.3015 & 0.3139 & 0.0123 & -0.0334 & -0.0336 & 0.0002 \\
\hline
\end{tabular}

\begin{tabular}{|c|c|c|c|c|c|c|}
\hline$\gamma=2$ & \multicolumn{3}{|c|}{ Volume } & \multicolumn{3}{c|}{ Kelvin lmpulse } \\
\cline { 2 - 7 } Time & B.I.M. & M.O.I. & Error & B.I.M. & M.O.I. & EnOT \\
\hline 0.0017 & 0.0047 & 0.0047 & 0.0000 & -0.0001 & -0.0001 & 0.0000 \\
0.0036 & 0.0116 & 0.0116 & 0.0000 & -0.0002 & -0.0002 & 0.0000 \\
0.0088 & 0.0339 & 0.0339 & 0.0000 & -0.0007 & -0.0007 & 0.0000 \\
0.0269 & 0.1275 & 0.1274 & 0.0000 & -0.0030 & -0.0030 & 0.0000 \\
0.1087 & 0.6447 & 0.6445 & 0.0002 & -0.0187 & -0.0188 & 0.0001 \\
0.4640 & 2.8705 & 2.8696 & 0.0009 & -0.0856 & -0.0860 & 0.0004 \\
1.1649 & 4.2146 & 4.2246 & 0.0099 & -0.1119 & -0.1130 & 0.0011 \\
1.7254 & 1.9013 & 1.9288 & 0.0275 & -0.1586 & -0.1638 & 0.0052 \\
1.9187 & 0.6385 & 0.6627 & 0.0242 & -0.1958 & -0.2003 & 0.0046 \\
\hline
\end{tabular}

Table 1 Numerical comparison between the two methods for the bubble volume and the Kelvin impulse.

remain near maximum bubble size while the bubble furthest away from the boundary collapses with a liquid jet directed towards the boundary. In Figure $4.1(\mathrm{c}), \gamma=2,4$ and 6. In this case we observe that the bubbles at $\gamma=2$ and 6 collapse first of all, with the liquid jets being directed towards the $\gamma=4$ bubble. In this case the central bubble "attracts" the bubble at $\gamma=2$ more strongly (see Kelvin impulse in Figure 4.2) than the rigid boundary because it is much closer. Thus, it would appear that bubbles will need to be very close to the boundary for the rigid boundary interaction to dominate. 

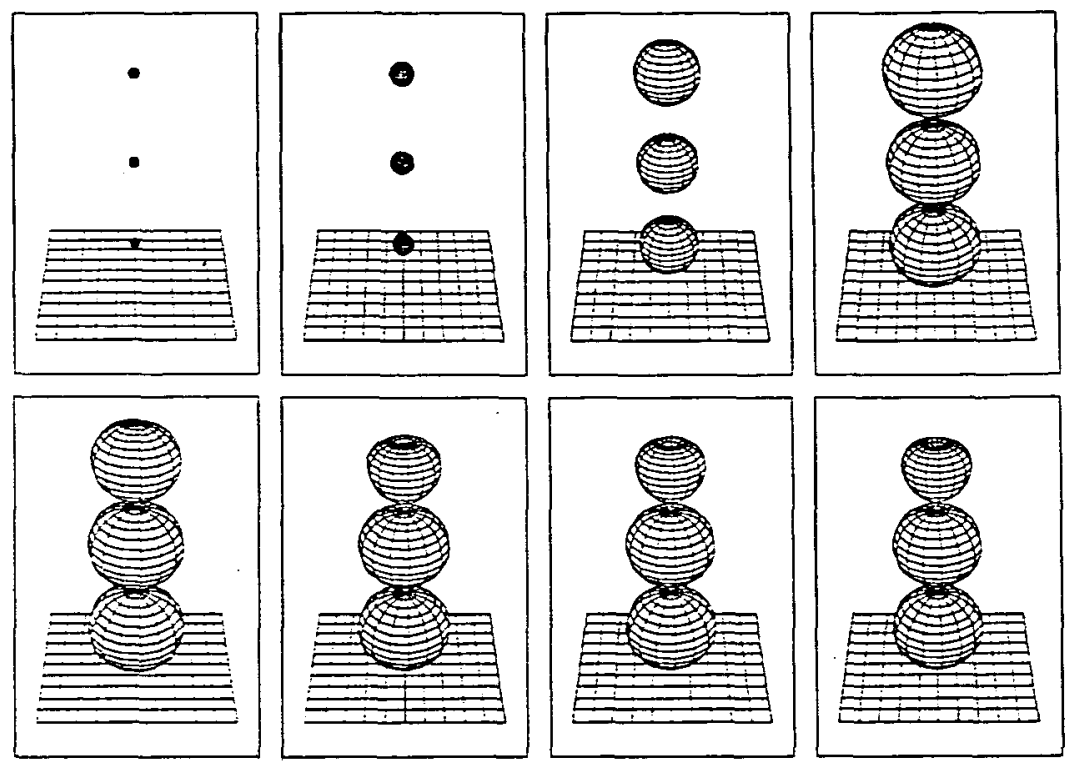

Figure 4.1(b) Three dimensional illustration of the growth and collapse of three bubbles initially located at $\gamma=1,3$ and 5 .
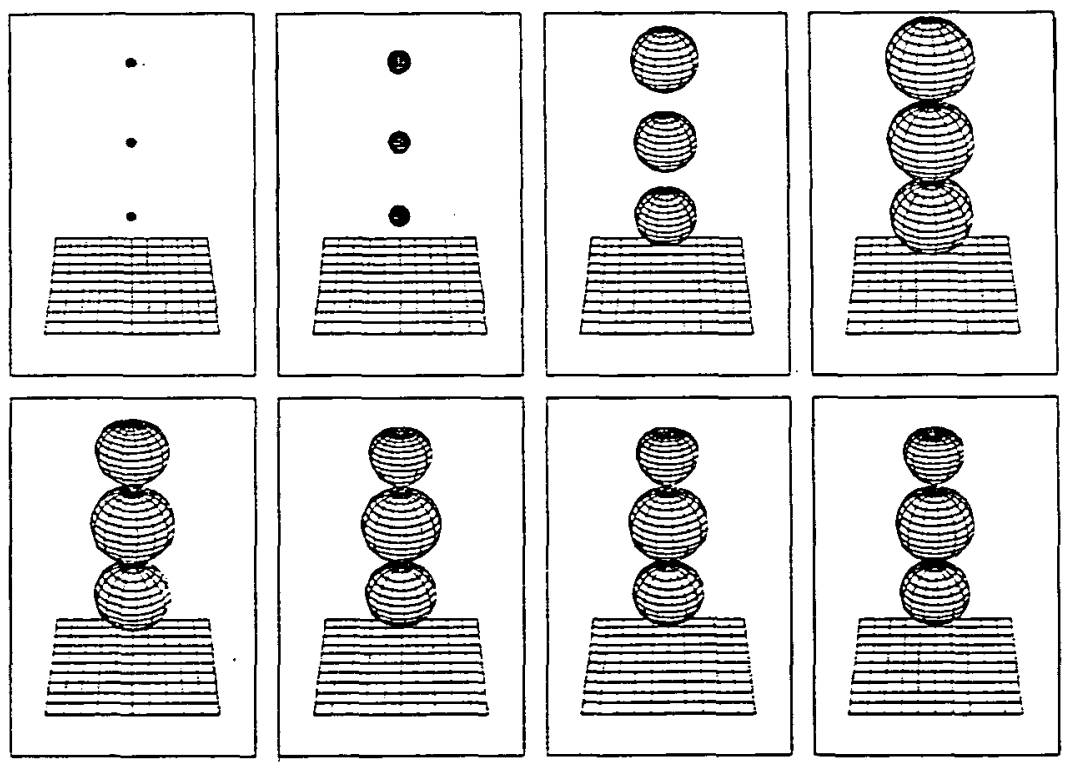

Figure 4.1(c) Three dimensional illustration of the growth and collapse of three bubbles initially located at $\gamma=2,4$ and 6 . 

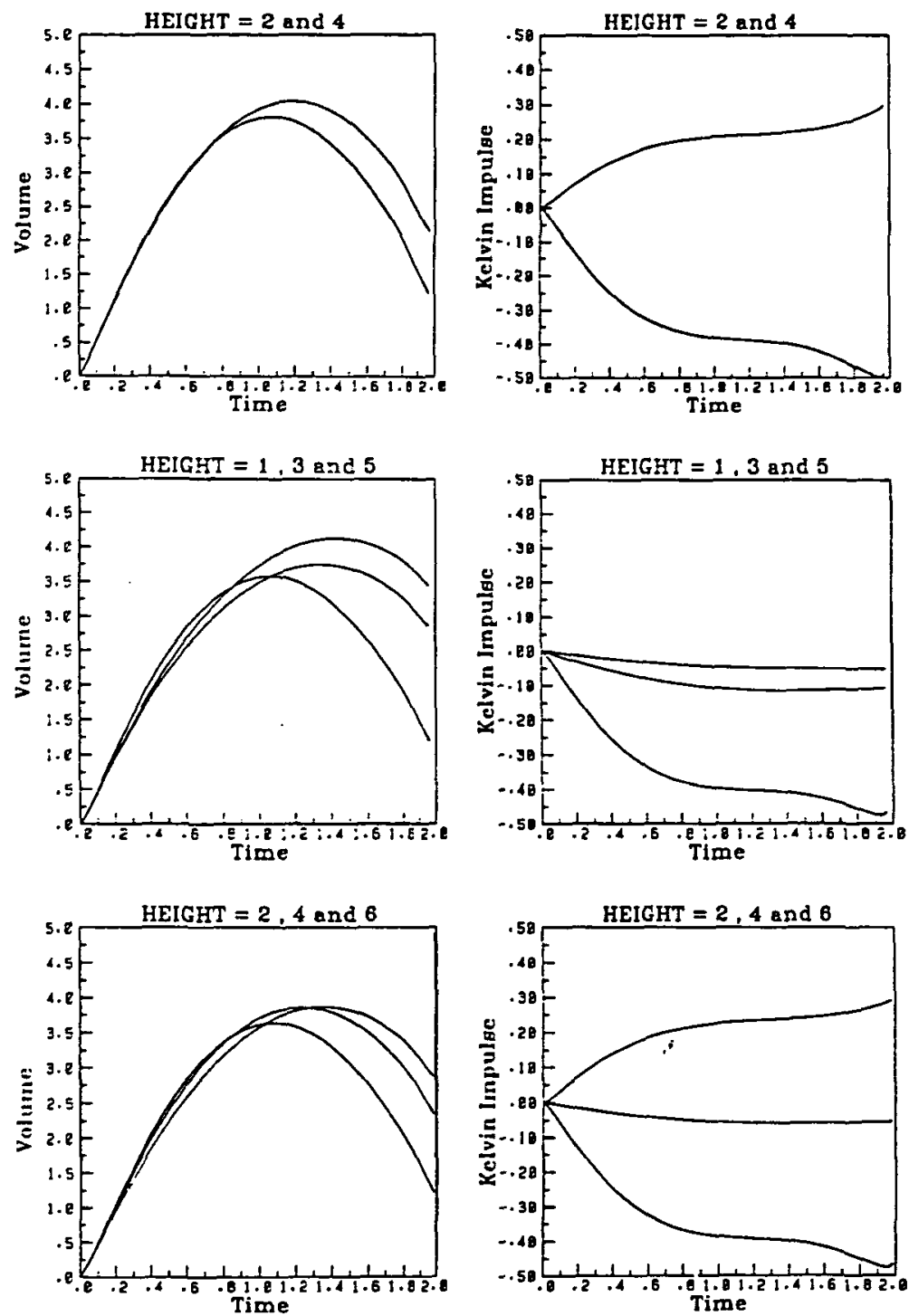

Figure 4.2 Continuous time comparisons of the bubble volumes and their Kelvin Impulses for bubbles in Figures 4.1(a), 4.1(b) and $4.1(\mathrm{c})$ respectively.

\section{NON-AXISYMMETRIC BUBBLES IN A SEMI-INFINITE REGION}

Non-axisymmetric bubbles in the semi-infinite region $(z \geq 0)$ will also be modelled using the method of images. Each bubble will be represented by a source, $m_{i}(t)$, and a 
dipole vector, $\left(d_{i}^{x}(t), d_{i}^{y}(t), d_{i}^{z}(t)\right)$, and their images in the $z=0$ plane (see Figure 5). The $\left(x_{i}, y_{i}, z_{i}\right)$ are the coordinates of the centroid of the $\mathrm{i}$-th bubble $(i=1,2, \cdots, N)$.

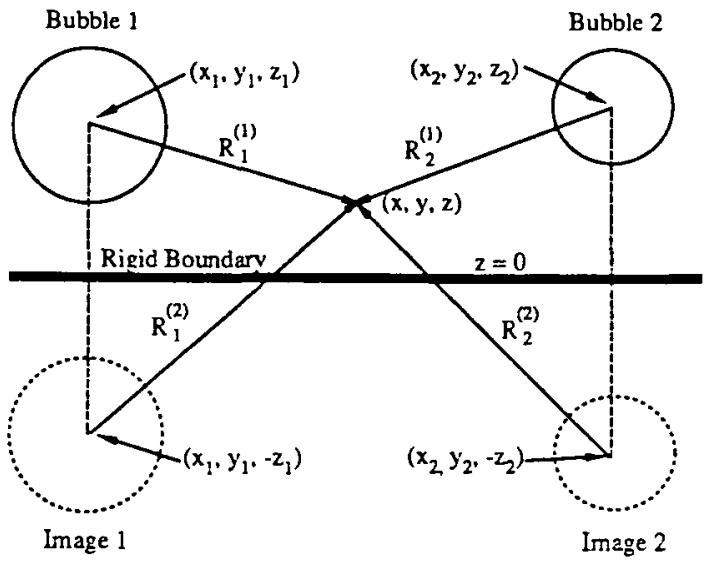

Figure 5 The geometry image systems for the case of nonaxisymmetric arrangements of bubble near an ifinite rigid boundary.

In this case the representation for each individual bubble is,

$$
\begin{aligned}
\phi_{i}=- & \frac{1}{4 \pi}\left\{m_{i}(t)\left[\frac{1}{R_{i}^{(1)}}+\frac{1}{R_{i}^{(2)}}\right]+d_{i}^{x}(t)\left[\frac{\left(x-x_{i}\right)}{R_{i}^{(1) 3}}+\frac{\left(x-x_{i}\right)}{R_{i}^{(2) 3}}\right]\right. \\
& \left.+d_{i}^{y}(t)\left[\frac{\left(y-y_{i}\right)}{R_{i}^{(1) 3}}+\frac{\left(y-y_{i}\right)}{R_{i}^{(2) 3}}\right]+d_{i}^{z}(t)\left[\frac{\left(z-z_{i}\right)}{R_{i}^{(1) 3}}-\frac{\left(z+z_{i}\right)}{R_{i}^{(2) 3}}\right]\right\},
\end{aligned}
$$

with

$$
\begin{aligned}
& R_{i}^{(1)}=\sqrt{\left(x-x_{i}\right)^{2}+\left(y-y_{1}\right)^{2}+\left(z-z_{i}\right)^{2}}, \\
& R_{i}^{(2)}=\sqrt{\left(x-x_{i}\right)^{2}+\left(y-y_{i}\right)^{2}+\left(z+z_{i}\right)^{2}} .
\end{aligned}
$$

Taking the gradient of (5.1) gives the velocity components due to this bubble in 
the $x, y$ and $z$ directions.

$$
\begin{aligned}
& u_{x}^{(i)}=\frac{\partial \phi_{i}}{\partial x}=\frac{-1}{4 \pi}\left\{\left(x-x_{i}\right) m_{i}(t)\left[\frac{1}{R_{i}^{(1) 3}}+\frac{1}{R_{i}^{(2) 3}}\right]\right. \\
& -d_{i}^{x}(t)\left[\left(\frac{1}{R_{i}^{(1) 3}}+\frac{1}{R_{i}^{(2) 3}}\right)-3\left(x-x_{i}\right)^{2}\left(\frac{1}{R_{i}^{(1) 5}}+\frac{1}{R_{i}^{(2) 5}}\right)\right] \\
& \left.-3\left(x-x_{i}\right) d_{i}^{y}(t)\left[\frac{\left(y-y_{i}\right)}{R_{i}^{(1) 5}}+\frac{\left(y-y_{i}\right)}{R_{i}^{(2) 5}}\right]-3\left(x-x_{i}\right) d_{i}^{z}(t)\left[\frac{\left(z-z_{i}\right)}{R_{i}^{(1) 5}}-\frac{\left(z+z_{i}\right)}{\dot{R}_{i}^{(2) 5}}\right]\right\} \text {, } \\
& u_{y}^{(i)}=\frac{\partial \phi_{i}}{\partial y}=\frac{-1}{4 \pi}\left\{\left(y-y_{i}\right) m_{i}(t)\left[\frac{1}{R_{i}^{(1) 3}}+\frac{1}{R_{i}^{(2) 3}}\right]\right. \\
& -3\left(y-y_{i}\right) d_{i}^{x}(t)\left[\frac{\left(x-x_{i}\right)}{R_{i}^{(1) 5}}+\frac{\left(x-x_{i}\right)}{R_{i}^{(2) 5}}\right] \\
& -d_{i}^{y}(t)\left[\left(\frac{1}{R_{i}^{(1) 3}}+\frac{1}{R_{i}^{(2) 3}}\right)-3\left(y-y_{i}\right)^{2}\left(\frac{1}{R_{i}^{(1) 5}}+\frac{1}{R_{i}^{(2) 5}}\right)\right] \\
& \left.-3\left(y-y_{i}\right) d_{i}^{z}(t)\left[\frac{\left(z-z_{i}\right)}{R_{i}^{(1) 5}}-\frac{\left(z+z_{i}\right)}{R_{i}^{(2) 5}}\right]\right\} \text {, } \\
& u_{z}^{(i)}=\frac{\partial \phi_{i}}{\partial z}=\frac{1}{4 \pi}\left\{-m_{i}(t)\left[\frac{\left(z-z_{i}\right)}{R_{i}^{(1) 3}}+\frac{\left(z+z_{i}\right)}{R_{i}^{(2) 3}}\right]-3\left(x-x_{i}\right) d_{i}^{x}(t)\left[\frac{\left(z-z_{i}\right)}{R_{i}^{(1) 5}}+\frac{\left(z+z_{i}\right)}{R_{i}^{(2) 5}}\right]\right. \\
& -3\left(y-y_{i}\right) d_{i}^{y}(t)\left[\frac{\left(z-z_{i}\right)}{R_{i}^{(1) 5}}+\frac{\left(z+z_{i}\right)}{R_{i}^{(2) 5}}\right]+d_{i}^{z}(t)\left[\left(\frac{1}{R_{i}^{(1) 3}}-\frac{1}{R_{i}^{(2) 3}}\right)\right. \\
& \left.\left.-3\left(\frac{\left(z-z_{i}\right)^{2}}{R_{i}^{(1) 5}}-\frac{\left(z+z_{i}\right)^{2}}{R_{i}^{(2) 5}}\right)\right]\right\} \text {. }
\end{aligned}
$$

Therefore the total velocity components in the $x, y$ and $z$ directions at any point due to the $N$ bubbles are given by

$$
u_{x}=\frac{\partial \phi}{\partial x}=\sum_{i=1}^{N} \frac{\partial \phi_{i}}{\partial x}, \quad u_{y}=\frac{\partial \phi}{\partial y}=\sum_{i=1}^{N} \frac{\partial \phi_{i}}{\partial y}, \quad u_{z}=\frac{\partial \phi}{\partial z}=\sum_{i=1}^{N} \frac{\partial \phi_{i}}{\partial z}
$$

As in the axisymmetric case, at each time step the individual centroids are updated using

$$
x_{i}=\frac{\int x d V_{i}}{\int d V_{i}}, \quad y_{i}=\frac{\int y d V_{i}}{\int d V_{i}}, \quad z_{i}=\frac{\int z d V_{i}}{\int d V_{i}}
$$

where the solution technique of Section 3 is employed to solve the system. 
(a)
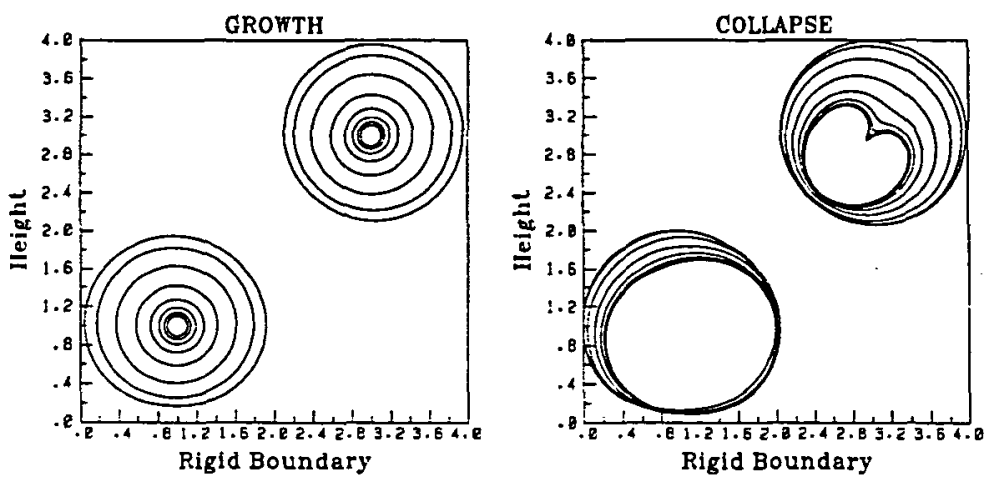

(b)
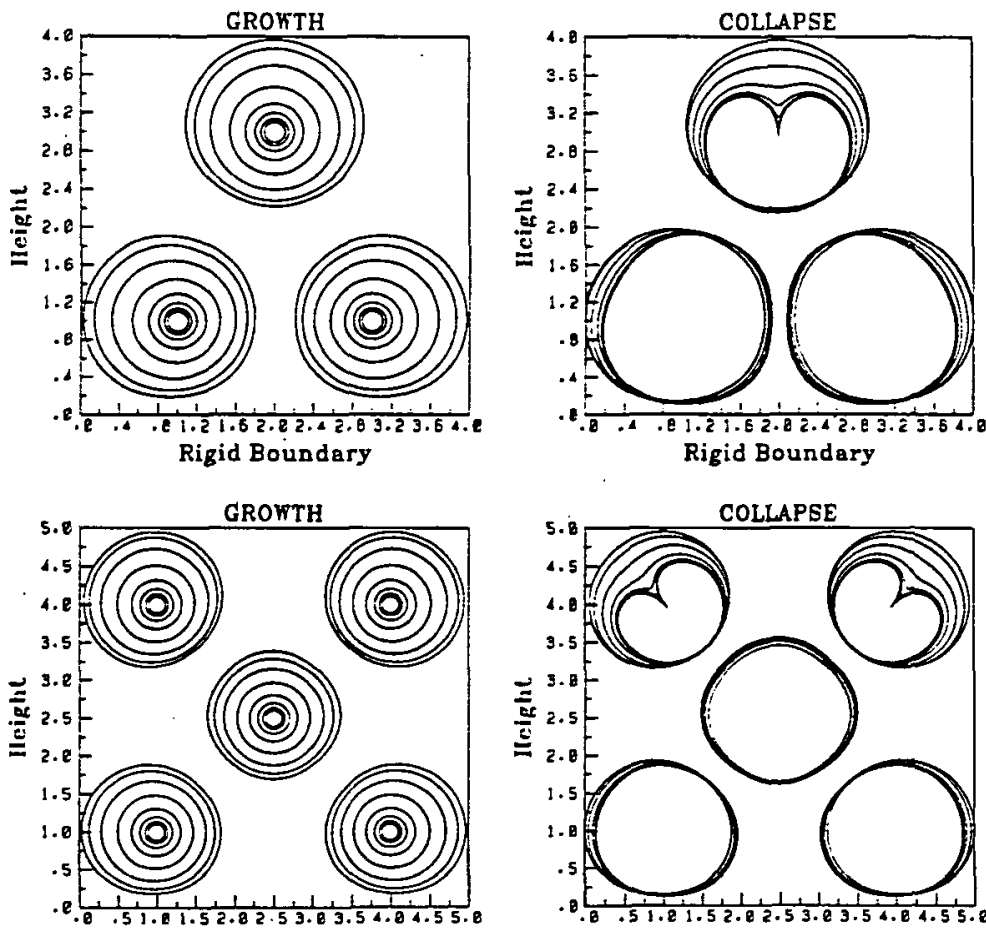

Rigid Boundary

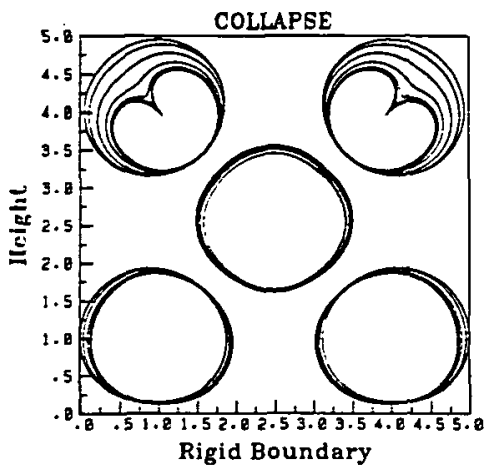

Figure 6 Examples of non-axisymmetric bubbles near a rigid boundary, showing the interactions with nearby bubbles and an adjacent rigid boundary.

Examples of our calculations are illustrated in Figure 6. In Figure 6(a) the initial coordinate location of the two bubbles is $(1,0,1)$ and $(3,0,3)$. It can be seen that both bubbles grow almost spherically. During the collapse phase, the bubble furthest away 
from the rigid boundary migrates towards the other bubble, developing a jet directed in this same direction. Indeed, because of the method of images, we might expect a behaviour not very dissimilar to that near a rigid boundary with a normal joining the line of centres of the two bubbles. The bubble closest to the boundary collapses at a slower rate under the influence of both the closer rigid boundary and the other bubble. As a result, the bubble collapses at an angle to the rigid boundary. In Figure 6(b), 3 bubbles with initial coordinates $(1,0,1),(2,0,3)$ and $(3,0,1)$ are considered. Because the bubbles are closer together in this case, there is a slight repulsion of the bubbles during the growth phase. We can interpret the collapse of the bubbles in terms of the planes of symmetry. The three planes that are midway between the bubbles with normals joining their initial line of centres will meet at the coordinate $(2, y, 2),|y|<\infty$. The uppermost bubble is therefore collapsing in an effectively wedge shaped geometry. Being furthest away from the boundary it collapses earlier than the other two. The two bubbles closest to the boundary collapse symmetrically, but at a much slower rate than the third bubble. In Figure 6(c), a similar arrangement to Figure 6(b) is considered but this time with 5 bubbles instead of 3 . Features of the previous two examples exist in this case. Two new features need to be identified in this case. Firstly the more advanced state of the outermost bubbles, and secondly the very slow collapse of the central bubble which is "shielded" on all sides by the other bubbles.

These calculations may have important implications for the practical case of clouds of cavitation bubbles near rigid boundaries. It suggests that a 'cascade' type phenomenon may occur with the outermost bubbles collapsing first of all, generating large pressures that may cause far higher collapse velocities in the bubbles closest to the boundary.

\section{BUBbLES IN CORNERS AND WEDGES}

The examples considered in the last section suggest we should also develop a theory for bubbles in corner and wedges. The method of images is easily extended to include bubble growth and collapse in a corner or a wedge (see Figures $7 \mathrm{a}$ and $7 \mathrm{~b}$ ). Following the same reasoning as in Sections 4 and 5 , the individual $\phi_{i}$ 's for the $90^{\circ}$ corner problem 


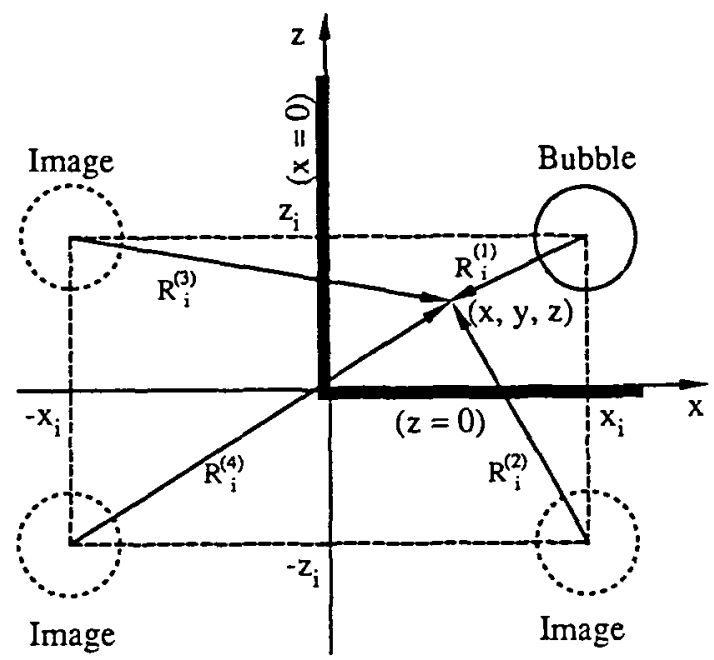

Figure $7(\mathrm{a})$ The image system for a bubble near a $90^{\circ}$ corner.

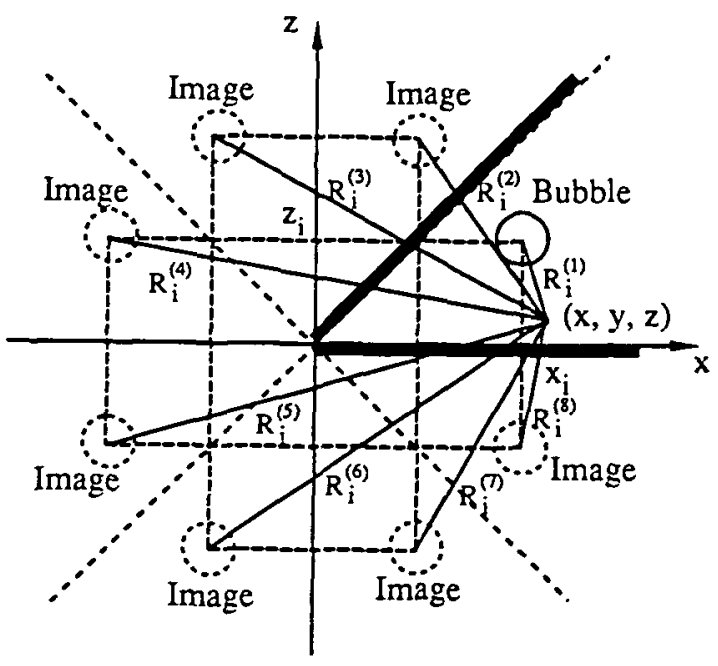

Figure $7(\mathrm{~b})$ The image system for a bubble near a $45^{\circ}$ corner. 
are given by

$$
\begin{aligned}
\phi_{i}=- & \frac{1}{4 \pi}\left\{m_{i}(t)\left[\frac{1}{R_{i}^{(1)}}+\frac{1}{R_{i}^{(2)}}+\frac{1}{R_{i}^{(3)}}+\frac{1}{R_{i}^{(4)}}\right]\right. \\
& +d_{i}^{z}(t)\left[\frac{\left(x-x_{i}\right)}{R_{i}^{(1) 3}}+\frac{\left(x-x_{i}\right)}{R_{i}^{(2) 3}}-\frac{\left(x+x_{i}\right)}{R_{i}^{(3) 3}}-\frac{\left(x+x_{i}\right)}{R_{i}^{(4) 3}}\right] \\
& +d_{i}^{y}(t)\left[\frac{\left(y-y_{i}\right)}{R_{i}^{(1) 3}}+\frac{\left(y-y_{i}\right)}{R_{i}^{(2) 3}}+\frac{\left(y-y_{i}\right)}{R_{i}^{(3) 3}}+\frac{\left(y-y_{i}\right)}{R_{i}^{(4) 3}}\right] \\
& \left.+d_{i}^{z}(t)\left[\frac{\left(z-z_{i}\right)}{R_{i}^{(1) 3}}-\frac{\left(z+z_{i}\right)}{R_{i}^{(2) 3}}+\frac{\left(z-z_{i}\right)}{R_{i}^{(3) 3}}-\frac{\left(z+z_{i}\right)}{R_{i}^{(4) 3}}\right]\right\},
\end{aligned}
$$

with

$$
\begin{aligned}
& R_{i}^{(1)}=\sqrt{\left(x-x_{i}\right)^{2}+\left(y-y_{i}\right)^{2}+\left(z-z_{i}\right)^{2}}, \\
& R_{i}^{(2)}=\sqrt{\left(x-x_{i}\right)^{2}+\left(y-y_{i}\right)^{2}+\left(z+z_{i}\right)^{2}}, \\
& R_{i}^{(3)}=\sqrt{\left(x+x_{i}\right)^{2}+\left(y-y_{i}\right)^{2}+\left(z-z_{i}\right)^{2}}, \\
& R_{i}^{(4)}=\sqrt{\left(x+x_{i}\right)^{2}+\left(y-y_{i}\right)^{2}+\left(z+z_{i}\right)^{2}},
\end{aligned}
$$

while the individual $\phi_{i}$ 's for the $45^{\circ}$ wedge problem are given by

$$
\begin{aligned}
\phi_{i}= & -\frac{1}{4 \pi}\left\{m_{i}(t)\left[\frac{1}{R_{i}^{(1)}}+\frac{1}{R_{i}^{(2)}}+\frac{1}{R_{i}^{(3)}}+\frac{1}{R_{i}^{(4)}}+\frac{1}{R_{i}^{(5)}}+\frac{1}{R_{i}^{(6)}}+\frac{1}{R_{i}^{(7)}}+\frac{1}{R_{i}^{(8)}}\right]\right. \\
+ & d_{i}^{x}(t)\left[\frac{\left(x-x_{i}\right)}{R_{i}^{(1) 3}}+\frac{\left(x-z_{i}\right)}{R_{i}^{(2) 3}}-\frac{\left(x+z_{i}\right)}{R_{i}^{(3) 3}}-\frac{\left(x+x_{i}\right)}{R_{i}^{(4) 3}}\right. \\
& \left.-\frac{\left(x+x_{i}\right)}{R_{i}^{(5) 3}}-\frac{\left(x+z_{i}\right)}{R_{i}^{(6) 3}}-\frac{\left(x-z_{i}\right)}{R_{i}^{(7) 3}}+\frac{\left(x-x_{i}\right)}{R_{i}^{(8) 3}}\right] \\
+ & d_{i}^{y}(t)\left[\frac{\left(y-y_{i}\right)}{R_{i}^{(1) 3}}+\frac{\left(y-y_{i}\right)}{R_{i}^{(2) 3}}+\frac{\left(y-y_{i}\right)}{R_{i}^{(3) 3}}+\frac{\left(y-y_{i}\right)}{R_{i}^{(4) 3}}\right. \\
& \left.+\frac{\left(y-y_{i}\right)}{R_{i}^{(5) 3}}+\frac{\left(y-y_{i}\right)}{R_{i}^{(6) 3}}+\frac{\left(y-y_{i}\right)}{R_{i}^{(7) 3}}+\frac{\left(y-y_{i}\right)}{R_{i}^{(8) 3}}\right] \\
+ & d_{i}^{z}(t)\left[\frac{\left(z-z_{i}\right)}{R_{i}^{(1) 3}}+\frac{\left(z-x_{i}\right)}{R_{i}^{(2) 3}}+\frac{\left(z-x_{i}\right)}{R_{i}^{(3) 3}}+\frac{\left(z-z_{i}\right)}{R_{i}^{(4) 3}}\right. \\
& -\frac{\left(z+z_{i}\right)}{\left.\left.R_{i}^{(5) 3}-\frac{\left(z+x_{i}\right)}{R_{i}^{(6) 3}}-\frac{\left(z+x_{i}\right)}{R_{i}^{(7) 3}}-\frac{\left(z+z_{i}\right)}{R_{i}^{(8) 3}}\right]\right\}}
\end{aligned}
$$


with

$$
\begin{aligned}
& R_{i}^{(1)}=\sqrt{\left(x-x_{i}\right)^{2}+\left(y-y_{i}\right)^{2}+\left(z-z_{i}\right)^{2}}, R_{i}^{(2)}=\sqrt{\left(x-z_{i}\right)^{2}+\left(y-y_{i}\right)^{2}+\left(z-x_{i}\right)^{2}}, \\
& R_{i}^{(3)}=\sqrt{\left(x+z_{i}\right)^{2}+\left(y-y_{i}\right)^{2}+\left(z-x_{i}\right)^{2}}, R_{i}^{(4)}=\sqrt{\left(x+x_{i}\right)^{2}+\left(y-y_{i}\right)^{2}+\left(z-z_{i}\right)^{2}}, \\
& R_{i}^{(5)}=\sqrt{\left(x+x_{i}\right)^{2}+\left(y-y_{i}\right)^{2}+\left(z+z_{i}\right)^{2}}, R_{i}^{(6)}=\sqrt{\left(x+z_{i}\right)^{2}+\left(y-y_{i}\right)^{2}+\left(z+x_{i}\right)^{2}}, \\
& R_{i}^{(7)}=\sqrt{\left(x-z_{i}\right)^{2}+\left(y-y_{i}\right)^{2}+\left(z+x_{i}\right)^{2}}, R_{i}^{(8)}=\sqrt{\left(x-x_{i}\right)^{2}+\left(y-y_{i}\right)^{2}+\left(z+z_{i}\right)^{2}} .
\end{aligned}
$$

The velocity components $\left(u_{x}, u_{y}, u_{z}\right)$ are obtained again by differentiating (6.1) for the corner problem and (6.3) for the wedge problem with respect to $x, y$ and $z$ respectively and summing the individual components (just as in Sections 4 and 5).

With the $\phi_{i}$ 's and $\left(u_{x}, u_{y}, u_{z}\right)$ known and the centroids $\left(x_{i}, y_{i}, z_{i}\right)$ calculated as given by (5.6) the solution procedure is identical to that given in Section 3.

Examples of the growth and collapse of cavitation bubbles in a $90^{\circ}$ corner are shown in Figure 8. In Figure 8(a) the growth and collapse of a single bubble symmetrically located at $(1.5,0,1.5)$ is considered. The growth is predominantly spherical and uninteresting. During the collapse phase the bubble migrates strongly towards the corner being under equal influence of the two rigid boundaries. In the second example, illustrated in Figure $8(\mathrm{~b})$, two bubbles located at $(1,0,3)$ and $(3,0,1)$ are considered. Again while the growth is predominantly spherical, there is a slight flattening of the bubble surfaces closest to the rigid boundaries. During the collapse phase the bubbles migrate and develop a jet towards the closest rigid boundary, although there is a slight inclination in the jet due to the presence of the other rigid boundary. In Figure 8(c) a more extensive problem is considered using 4 bubbles. Perhaps the most interesting aspect of this particular calculation is the almost total collapse of the bubble furthermost away from the corner before the other three have barely begun their collapse phase. Again strong evidence of the cascade of collapse phenomena is exhibited.

In Figure 9 further examples of the collapse of one, two and three bubbles in a $45^{\circ}$ wedge are presented. In all cases the growth phase is almost spherical with predominant collapse towards the nearest rigid boundary but modified by the presence of adjacent bubbles and the other rigid boundary. The bubble right in the corner collapses extremely slowly because it can only draw liquid in from the sides.

\section{BUbBLES NEXT TO A SEMI-INFINITE PLANE}

The ordinary method of images, as employed in Sections 4, 5 and 6, can only be used when the bounding planes meet at an angle $\pi / m(m=1,2, \ldots)$, but it fails when the angle of inclination is $k \pi / m$ (with $k$ and $m$ being positive and relatively 
(a)
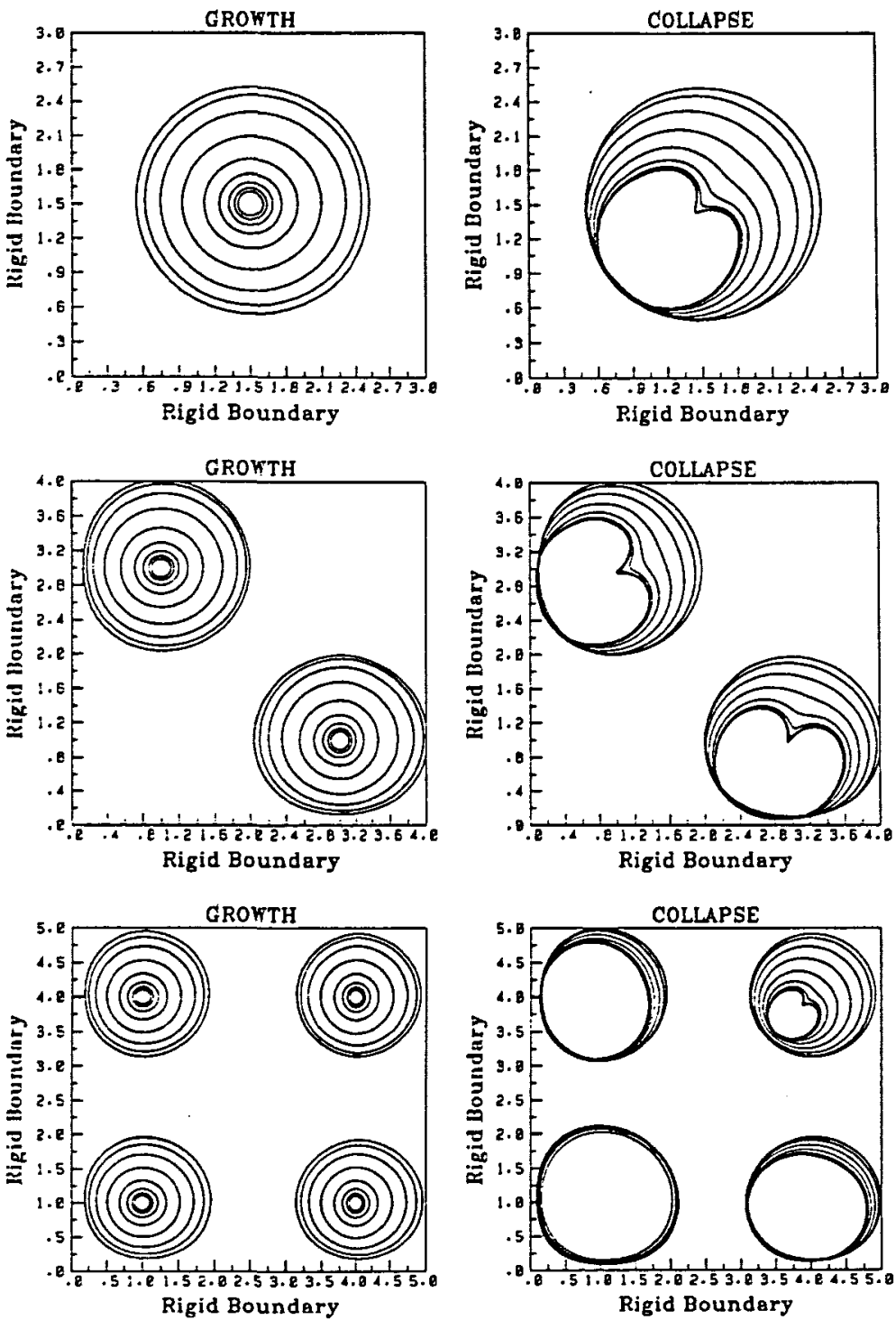

Figure $8 \mathrm{Growth}$ and collapse of bubbles in a $90^{\circ}$ corner. In (a) the initial coordinate location is $(1.5,1.5)$; in (b) $(1,3)$ and $(3,1)$ and in $(c)(1,1)(1,4),(4,1)$ and $(4.4)$.

prime integers). The trouble lies in the fact that reflection of the original source in the bounding planes generates images in the desired region. However, the problem can be overcome by solving Equation (2.1) not on a ordinary space but rather on the 
(a)
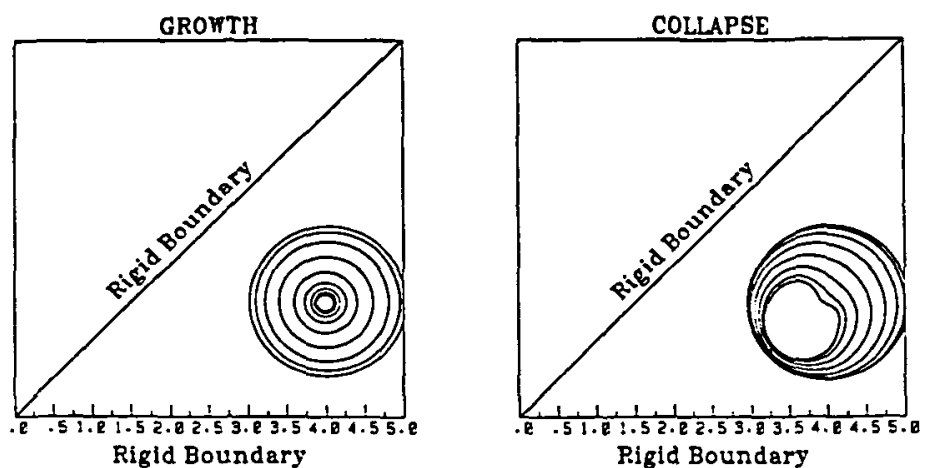

GROWTH
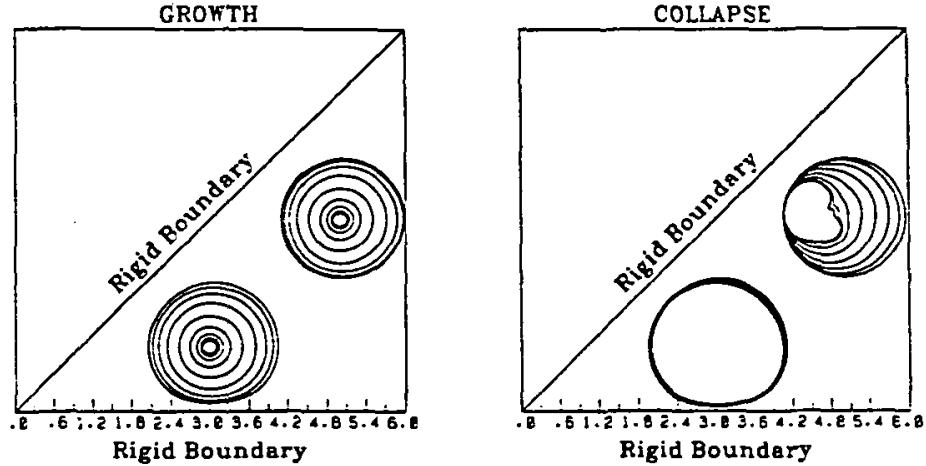

GROWTH

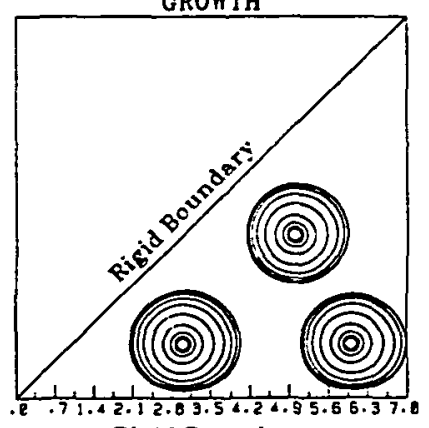

Rigid Boundery

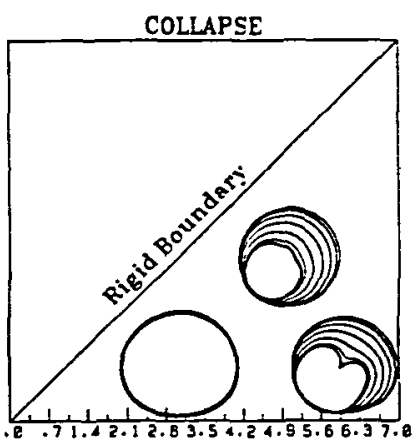

Rigid Boundary

Figure 9 Growth and collapse of bubbles in a $45^{\circ}$ corner. Initial coordinate locations in (a) are $(4.0,1.5)$; in (b) $(1,3)$ and $(3,1)$ and in $(c)(1,1)(1,4),(4,1)$ and $(4.4)$.

appropriate Riemann space. This method was first proposed by Sommerfield [20] and is analogous to the Riemann surface of the theory of functions of a complex, variable. Thus multivalued functions become single valued in the Riemann space. 


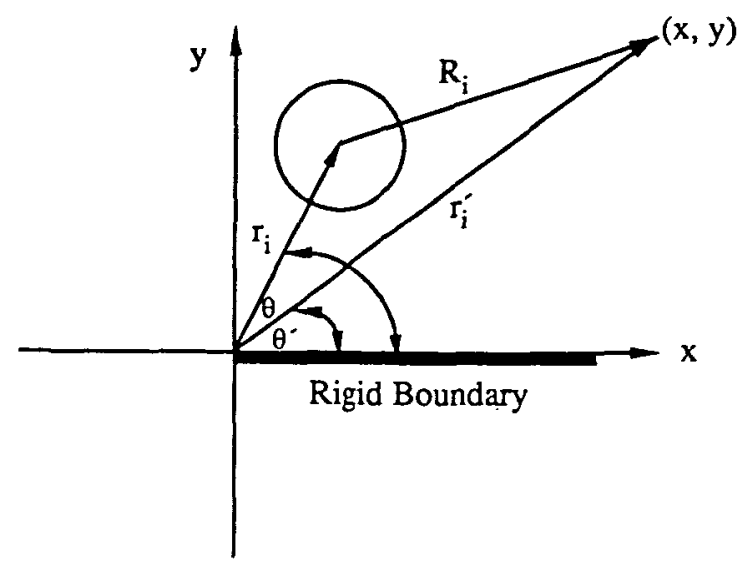

Figure 10 The coordinates and notation for a semi-infinite rigid boundary.

For the semi-infinite rigid boundary (see Figure 10) if one takes the range $0<\theta<$ $2 \pi$ to denote the physical space; and $-2 \pi<\theta<0$ for the "imaginary" space, one can build up the required two fold Riemann space, with the appropriate potential given by

$$
\phi_{i}=m_{i}(t)\left\{\frac{2}{\pi R_{i}^{(1)}} \arctan \sqrt{\frac{\sigma+\tau}{\sigma-\tau}}+\frac{2}{\pi R_{i}^{(2)}} \arctan \sqrt{\frac{\sigma+\tau^{\prime}}{\sigma-\tau^{\prime}}}\right\},
$$

with

$$
\begin{aligned}
& R_{i}^{(1)}=\sqrt{r_{i}^{2}+r_{i}^{\prime 2}-2 r_{i} r_{i}^{\prime} \cos \left(\theta_{i}-\theta_{i}^{\prime}\right)+\left(z-z_{i}\right)^{2}}, \\
& R_{i}^{(2)}=\sqrt{r_{i}^{2}+r_{i}^{\prime 2}-2 r_{i} r_{i}^{\prime} \cos \left(\theta_{i}+\theta_{i}^{\prime}\right)+\left(z-z_{i}\right)^{2}},
\end{aligned}
$$

and

$$
\begin{gathered}
\tau=\cos \left(\frac{\theta_{i}-\theta_{i}^{\prime}}{2}\right), \quad \tau^{\prime}=\cos \left(\frac{\theta_{i}+\theta_{i}^{\prime}}{2}\right) \\
2 r r^{\prime} \cosh \alpha_{i}=r_{i}^{2}+r_{i}^{\prime 2}+\left(z-z_{i}\right)^{2}, \quad \sigma=\cosh \left(\frac{\alpha_{i}}{2}\right) .
\end{gathered}
$$

Differentiating the source solution (7.1) numerically with respect to $x, y$ and $z$ generates the required dipoles, after which the solution technique is identical to the previous cases.

In Figure 11.1 several tests of the theory are conducted. In Figure 11.1(a), the initial bubble centroid is in the same plane as the semi-infinite boundary. The bubble grows and collapses like a bubble in an infinite fluid, as one might expect. The next 
(a)
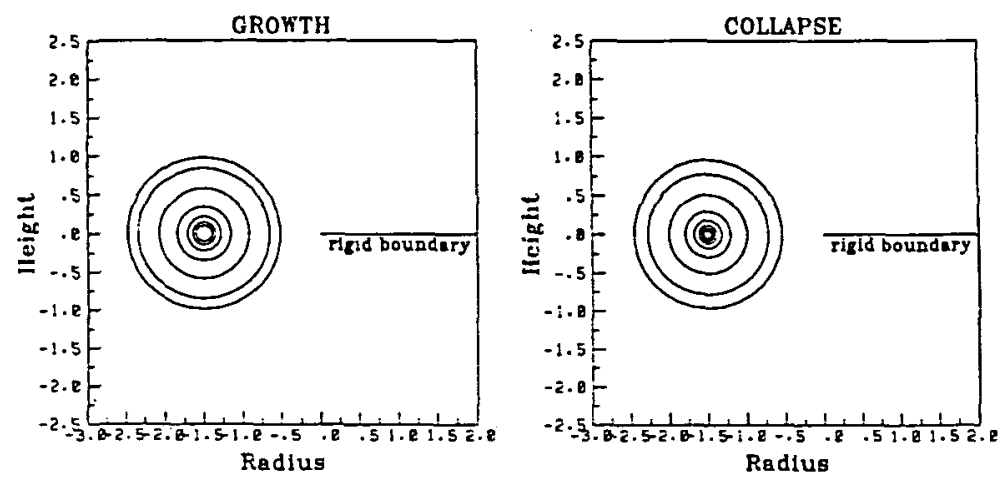

(b)
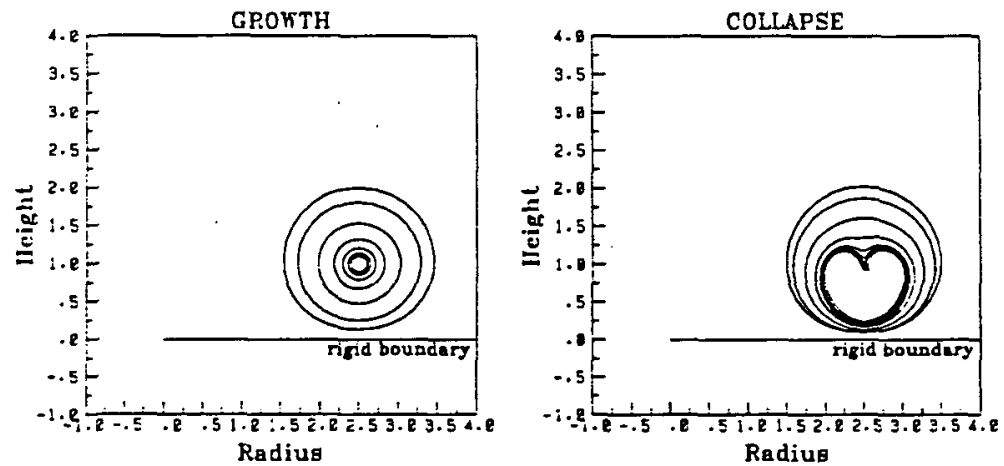

(c)
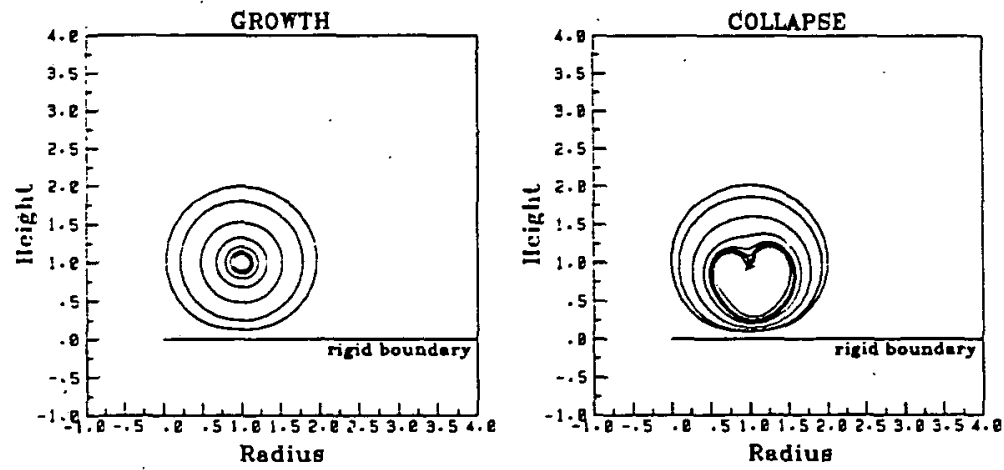

Figure 11.1 Growth and collapse of a cavitation bubble near a semi-infinite rigid boundary. The influence of the edge becomes apparent in (c).

example in Figure 11.1(b) is for a bubble 2.5 units along the rigid boundary from the edge. In this case the bubble grows and collapses as if it is near an infinite rigid boundary, the liquid jet being directed towards the boundary Figure 11.1(c) considers 
the case of a bubble much closer to the edge, in this case only one unit along the rigid boundary. While the growth phase is almost spherical, the direction of jet formation is tilted away slightly from the edge. This is because it is easier for the bubble to "draw-in" liquid from this direction.

Figure 11.2 considers several multi-bubble examples near the leading edge. In Figure 11.2(a) the two bubbles are initially located at $(0.5,0,10)$ and $(2.5,0,1.0)$. During the collapse phase, the bubble closest to the leading edge collapses marginally faster because it has more 'free space' from which to "draw-in" liquid. Otherwise it is relatively symmetric. The initial coordinates in Figure $11.2(\mathrm{~b})$ are $(-1.5,0,1.0)$ and $(0.5,0,1.0)$. In this case, the bubble clear from the leading edge migrates and collapses towards the other bubble. The bubble closest to the edge appears to develop two jets, one being associated with the ease of liquid flow around the leading edge, although this may also be associated with the singularity at the leading edge. Figure 11.2(c) displays the symmetries of the 3-bubble problem that have been discussed earlier although, again, the leading edge distorts the bubble collapse to a slight extent.

\section{BubBles IN CIRCULAR PIPES}

The study of the problem of a cavitation bubble in a circular pipe is of great interest because Reynold's original experiments were conducted in a cylindrical tube. In addition nuclei-counter devices are based on cylindrical geometries. The theory discussed in this section will incorporate a number of important facets of this problem.

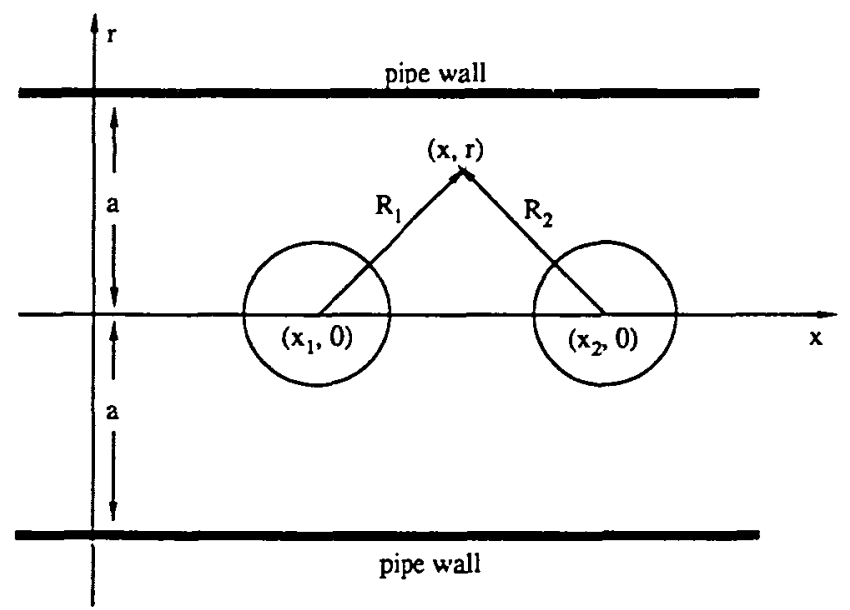

Figure 12 The coordinate system for the Green's functions in a circular cylinder. 
(a)
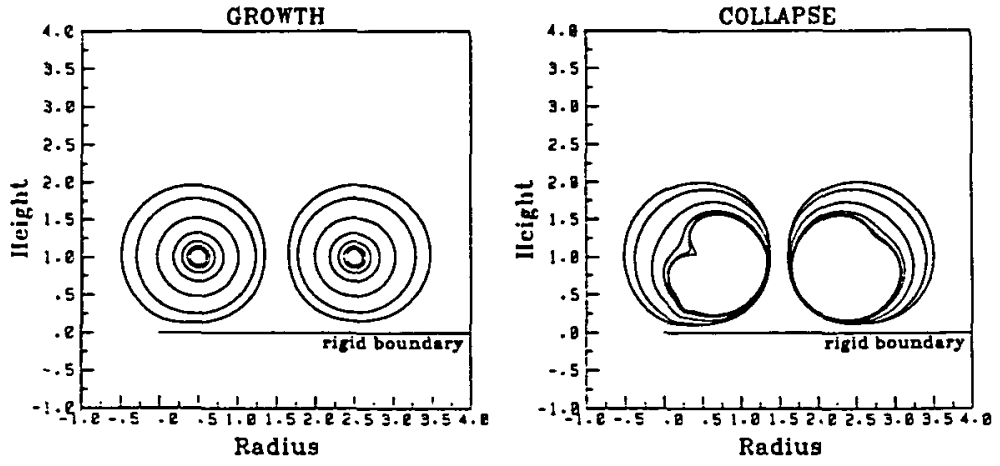

(b)
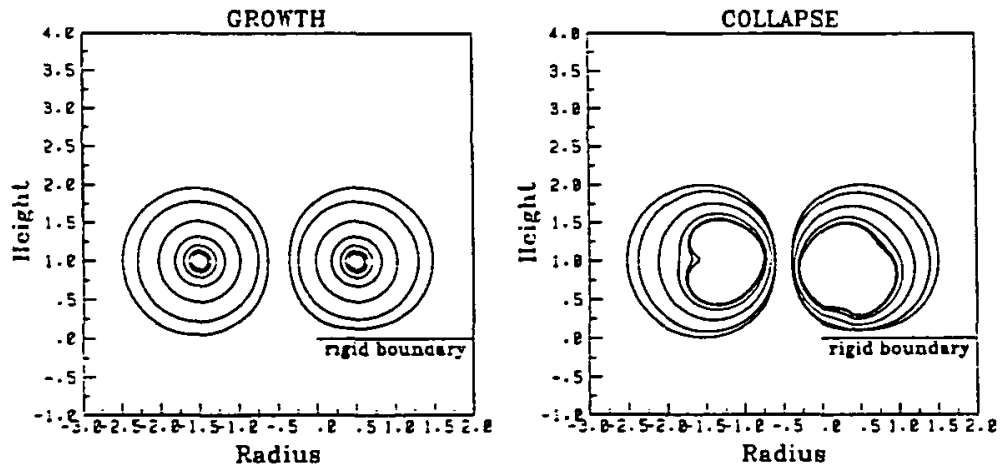

(c)
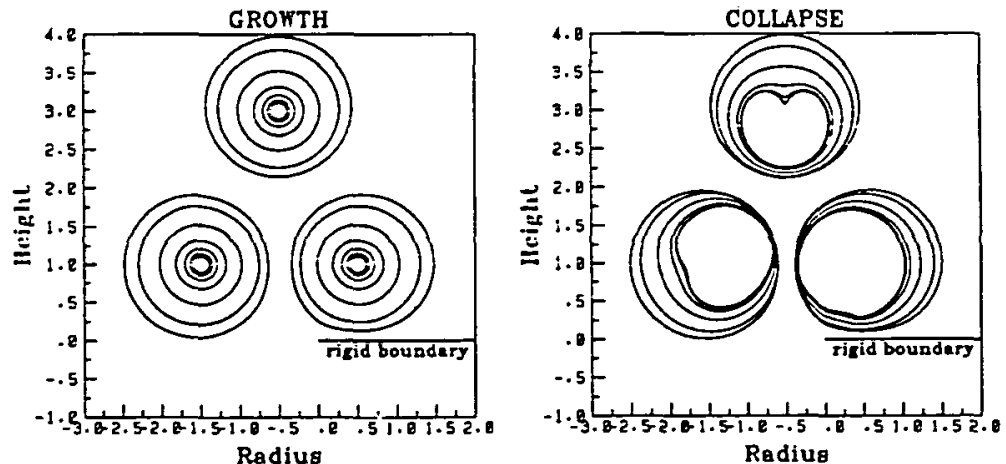

Figure 11.2 The growth and collapse of 2 and 3 bubbles near the edge of a semi-infinite boundary. Initial coordinate locations are $(0.5,1)$ and $(2.5,1) ;(b)(-1.5,1)$ and $(0.5,1)$ and $(c)$ $(-1.5,1),(0.5,1)$ and $(-0.5,3)$.

Employing cylindrical coordinates $(x, r, \theta)$, with the circular pipe of radius $a$ (see 
Figure 12) the individual $\phi_{i}$ 's are given by the axisymmetric Green's function

$$
\begin{array}{r}
\phi_{i}=m_{i}(t)\left\{\frac{-1}{R_{i}}+\frac{2}{\pi a} \int_{0}^{\infty} \frac{K_{1}(\xi)}{I_{1}(\xi)}\left[I_{0}(\xi)-I_{0}(\xi r / a) \cos \left[\xi\left(x-x_{i}\right) / a\right]\right] d \xi\right\} \\
+d_{i}^{x}(t)\left\{\frac{\left(x-x_{i}\right)}{R_{i}^{3}}+\frac{2}{\pi a} \int_{0}^{\infty} \frac{K_{1}(\xi)}{I_{1}(\xi)} I_{0}(\xi r / a) \sin \left[\xi\left(x-x_{i}\right) / a\right] d \xi\right\},
\end{array}
$$

where $K_{1}$ is a modified Bessel function of the third kind, $I_{0}$ and $I_{1}$ are modified Bessel functions of the second kind, and the $R_{i}$ are given by

$$
R_{i}=\sqrt{\left(x-x_{i}\right)^{2}+r^{2}}
$$

For a detailed discussion and derivation of (8.1) see Landweber [10].

Differentiating (8.1) with respect to $x$ and $r$ gives the velocities

$$
\begin{aligned}
u_{x}^{(i)}=\frac{\partial \phi_{i}}{\partial x}= & m_{i}(t)\left\{\frac{\left(x-x_{i}\right)}{R_{i}^{3}}+\frac{2}{\pi a^{2}} \int_{0}^{\infty} \frac{\xi K_{1}(\xi)}{I_{1}(\xi)} I_{0}(\xi r / a) \sin \left[\xi\left(x-x_{i}\right) / a\right] d \xi\right\} \\
& +d_{i}^{x}(t)\left\{\frac{1}{R_{i}^{3}}-\frac{3\left(x-x_{i}\right)^{2}}{R_{i}^{5}}\right. \\
& \left.+\frac{2}{\pi a^{3}} \int_{0}^{\infty} \frac{\xi^{2} K_{1}(\xi)}{I_{1}(\xi)} I_{0}(\xi r / a) \cos \left[\xi\left(x-x_{i}\right) / a\right] d \xi\right\}, \\
u_{r}^{(i)}=\frac{\partial \phi_{i}}{\partial r}= & m_{i}(t)\left\{\frac{r}{R_{i}^{3}}-\frac{2}{\pi a^{2}} \int_{0}^{\infty} \frac{\xi K_{1}(\xi)}{I_{1}(\xi)} I_{1}(\xi r / a) \cos \left[\xi\left(x-x_{i}\right) / a\right] d \xi\right\} \\
& +d_{i}^{x}(t)\left\{-\frac{3 r\left(x-x_{i}\right)}{R_{i}^{5}}+\frac{2}{\pi_{a}^{3}} \int_{0}^{\infty} \frac{\xi^{2} K_{1}(\xi)}{I_{1}(\xi)} I_{1}(\xi r / a) \sin \left[\xi\left(x-x_{i}\right) / a\right] d \xi\right\}
\end{aligned}
$$

Results of our calculations in cylindrical geometries may be found in Figures 13, 14.1, 14.2 and 15. In Figure 13 the growth and collapse of a single bubble is considered for the cases when the dimensionless radius of the cylinder is 2.0,1.5 and 1.0 in Figures 13(a), (b) and (c) respectively. During the growth phase the bubbles become more elongated as the radius of the cylinder decreases. In the collapse phase it is easier to "draw-in" liquid from along the axis, yielding two equal and opposite liquid jets which will lead to a toroidal shaped bubble. Figure 14.1 considers the example of multibubbles along the axis of a cylinder. In Figures 14.1(a) and (b), we can imagine that a plane boundary exists midway between the two bubbles because of the method of images. The interesting feature of both these examples is the significant migration of the bubbles during collapse. This is because there will always be net outflow or inflow along the entire cylinder, depending on whether the bubble is expanding or collapsing. Thus the growth and collapse of a bubble will be felt at infinity as much as close to 
(a)
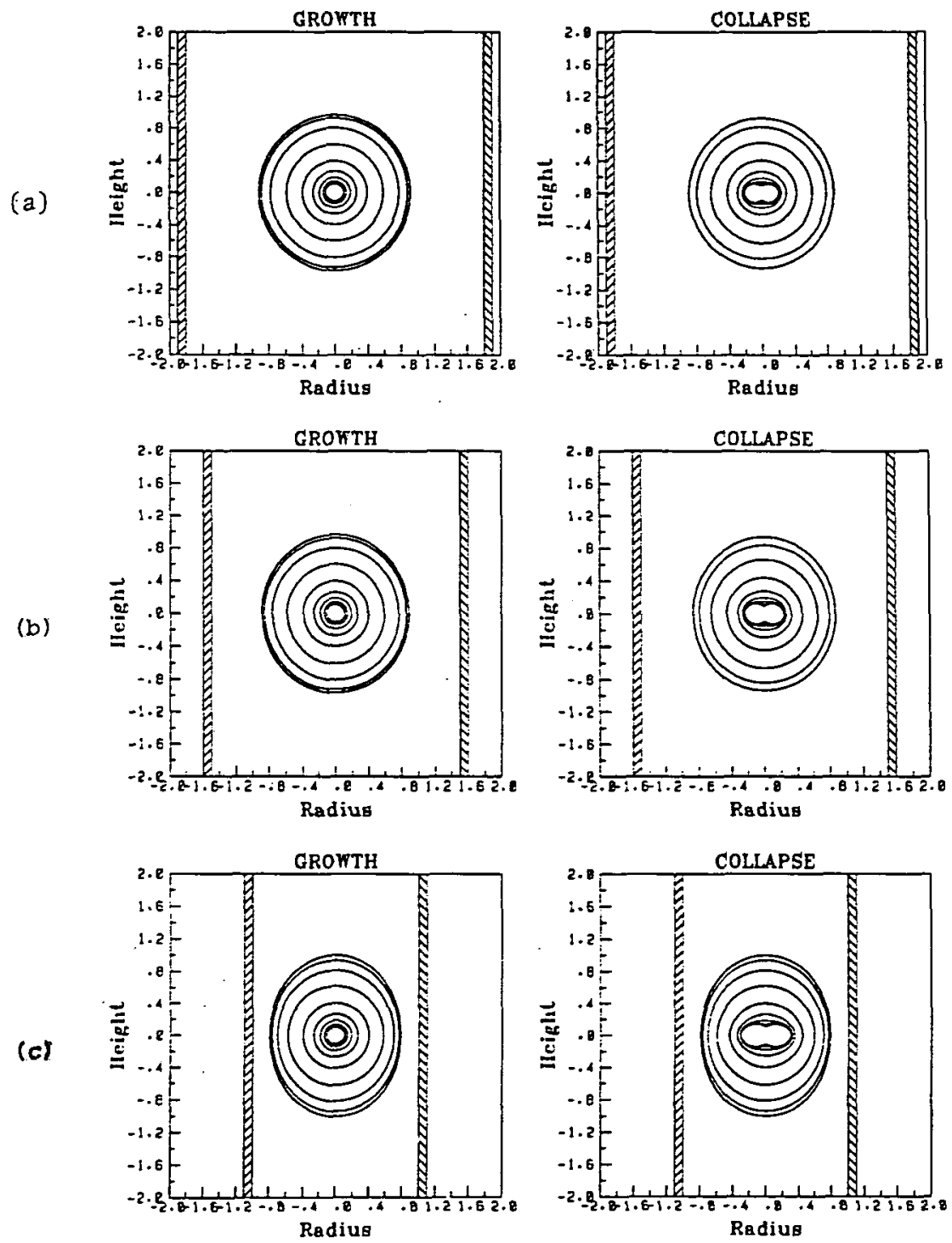

Figure 13 Growth and collapse of a single bubble in a circular pipe. The dimensionless radius of the sylinder in (a) is 2.0 , (b) 1.5 and (c) 1.0. Symmetric jets develop along the axis in all cases.

the bubble. Figure 14.1(c) considers the 3 bubble example with the outermost two bubbles collapsing far earlier than the central bubble. The description of the bubble 
shape is further emphasised by the calculations on the volume and the Kelvin-impulse (see Figure 14.2). In the symmetric bubble case in the 3 figures the calculated Kelvin impulse are equal in magnitude and opposite in sign, whereas the central bubble in Figure 14.2(c) has a zero impulse because of symmetry. Three dimensional illustrations of the bubble shape are shown in Figure 15(a) and (b) for the Figure 14.1(a) and (c) examples. These illustrations give a much better impression of the actual size of the bubbles than the more common two-dimensional sections.
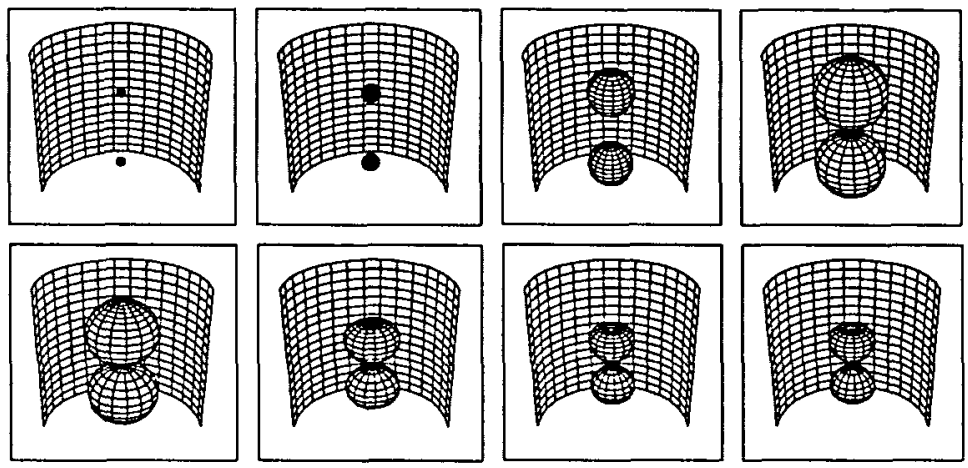

Figure 15(a) Three dimensional computer graphics illustration of Figure 14.1(a).
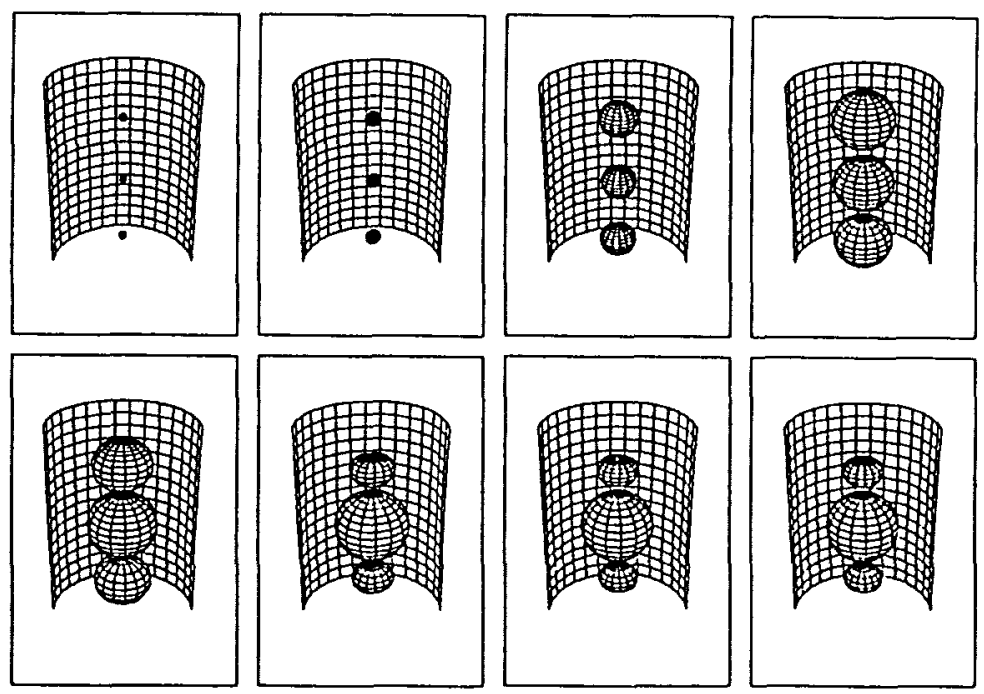

Figure 15(b) Three dimensional computer illustrations of Figure 14.1(c), especially emphasising the large volume differences. 

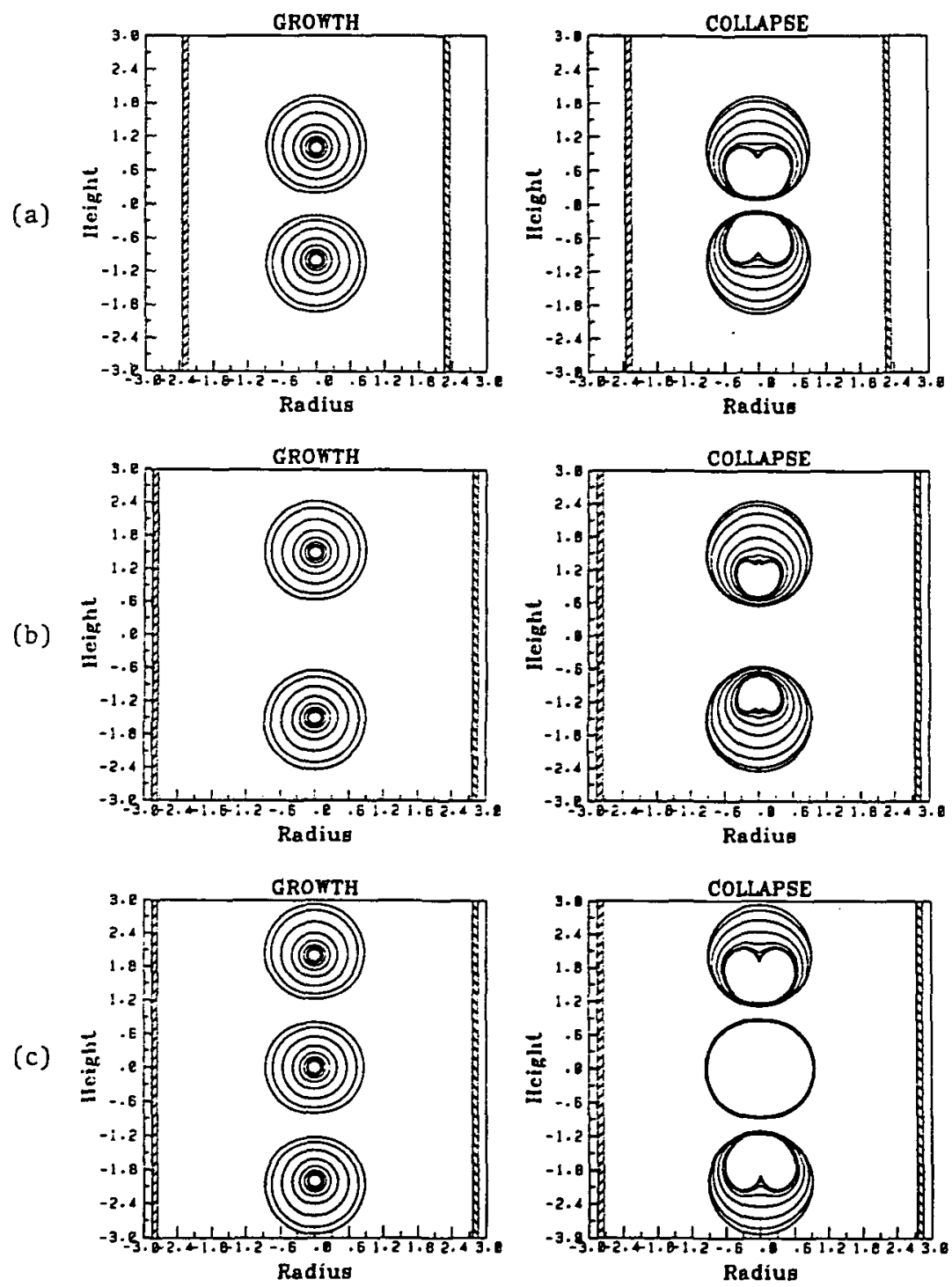

Figure 14.1 Growth and collapse of 2 and 3 bubbles along the line of axis of the cylinder.

\section{BubBles BetweEn parallel plates}

Employing $(x, r)$ coordinates, with the plate separation $H$ (see Figure 16), the 

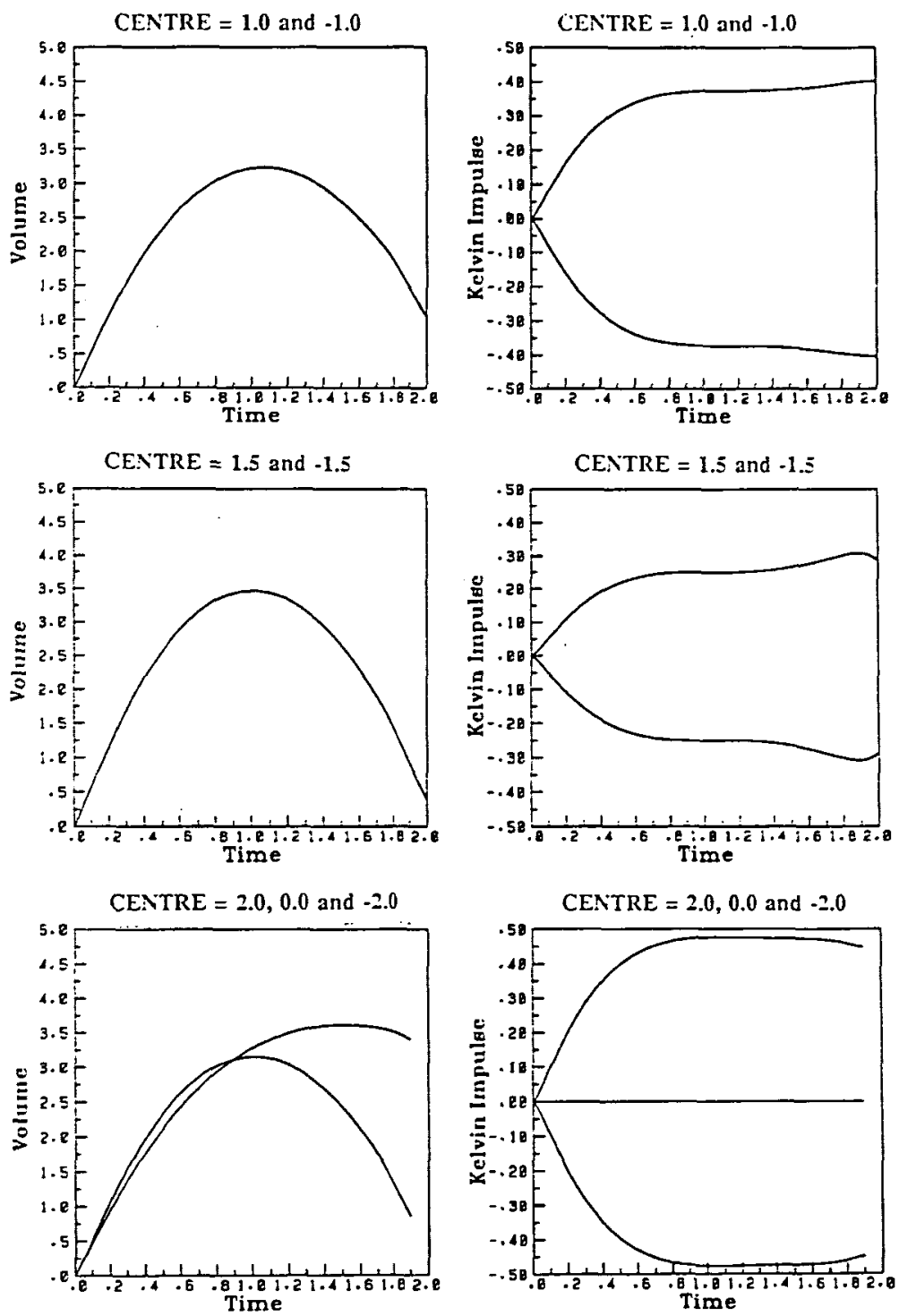

Figure 14.2 Continuous time comparisons of the bubble volumes and their Kelvin Impulses for bubbles in Figures 14.1(a), $14.1(\mathrm{~b})$ and $14.1(\mathrm{c})$ respectively. 
individual $\phi_{i}$ 's are given by the Hankel transforms

(9.1)

$$
\begin{aligned}
& \phi_{i}=\frac{1}{4 \pi}\left\{m _ { i } ( t ) \left[\frac{1}{R_{i}}\right.\right. \\
& \left.+\int_{0}^{\infty} \frac{\left[\left(\cosh \xi x J_{0}(\xi r)-\cosh \xi H\right) e^{-\left(H-x_{i}\right) \xi}+\left(\cosh [(H-x) \xi] J_{0}(\xi r)-\cosh \xi H\right) e^{-\xi x_{i}}\right]}{\sinh \xi H} d \xi\right] \\
& +d_{i}^{x}(t)\left[\frac{\left(x-x_{i}\right)}{R_{i}^{3}}\right. \\
& \left.\left.+\int_{0}^{\infty} \xi \frac{\left[\left(\cosh \xi x J_{0}(\xi r)-\cosh \xi H\right) e^{-\left(H-x_{i}\right) \xi}-\left(\cosh [(H-x) \xi] J_{0}(\xi r)-\cosh \xi H\right) e^{-\xi x_{i}}\right]}{\sinh \xi H} d \xi\right]\right\}
\end{aligned}
$$

where $J_{0}$ is the Bessel function of the first kind, zeroth order, and $R_{i}$ are given by

$$
R_{i}=\sqrt{\left(x-x_{i}\right)^{2}+r^{2}}
$$

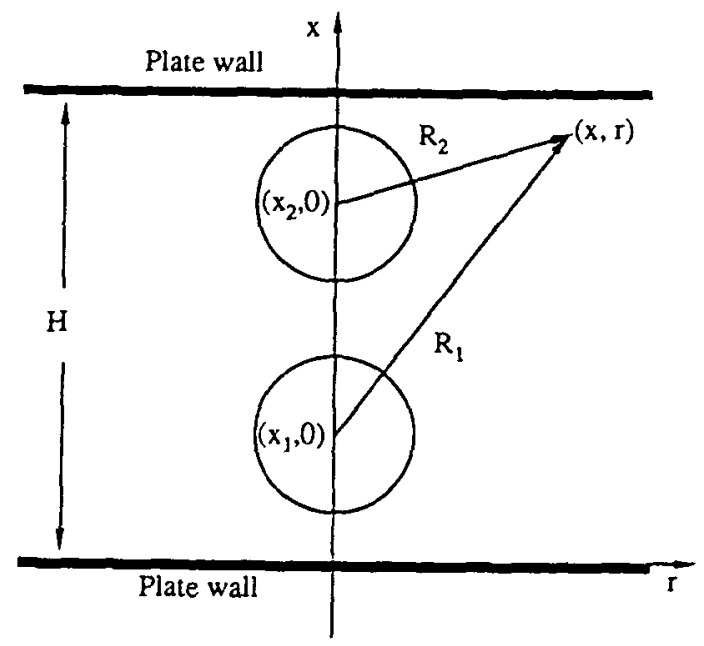

Figure 16 The coordinate system for the Green's function for two parallel plates.

The $\cosh \xi H$ terms in the integrands of Equation (9.1) had to be introduced to eliminate the $\xi^{-1}$ singularities at $\xi=0$ present from the $\sinh \xi H$ denominator term. The added terms, being independent of $x$ and $r$, serve only as constants, and do not effect the solution. At first glance, there does appear to be a certain amount of "freedom" as to the particular form of the introduced terms. For example, if one only took the limit as $\xi \rightarrow 0$ of $\cosh \xi x J_{0}(\xi r)$ and $\cosh [(H-x) \xi] J_{0}(\xi r)$ one could be tempted to try 
$\left(\cosh \xi x J_{0}(\xi r)-1\right)$ and $\left(\cosh [(H-x) \xi] J_{0}(\xi r)-1\right)$. However, although the singularities would be extracted, the symmetry would be destroyed. For example, if $x_{i}=H / 2$ (a single bubble in the middle of two plates) the potential at $x=x_{i}+\varepsilon$ would not be equal to the potential at $x=x_{i}-\varepsilon$.

Differentiating (9.1) with respect to $r$ and $x$ gives the following velocities

$$
\begin{aligned}
u_{r}^{(i)}= & \frac{\partial \phi_{i}}{\partial r}=\frac{-1}{4 \pi}\left\{m _ { i } ( t ) \left[\frac{r}{R_{i}^{3}}\right.\right. \\
& \left.+\int_{0}^{\infty} \xi \frac{\left[\cosh \xi x e^{-\left(H-x_{i}\right) \xi}+\cosh [(H-x) \xi] e^{-\xi x_{i}}\right]}{\sinh \xi H} J_{1}(\xi r) d \xi\right] \\
& +d_{i}^{x}(t)\left[\frac{\left(x-x_{i}\right) r}{R_{i}^{5}}\right. \\
& \left.\left.+\int_{0}^{\infty} \xi^{2} \frac{\left[\cosh \xi x e^{-\left(H-x_{i}\right) \xi}-\cosh [(H-x) \xi] e^{-\xi x_{i}}\right]}{\sinh \xi H} J_{1}(\xi r) d \xi\right]\right\}, \\
u_{x}^{(i)}= & \frac{\partial \phi}{\partial x}=\frac{1}{4 \pi}\left\{m _ { i } ( t ) \left[\frac{-\left(x-x_{i}\right)}{R_{i}^{3}}\right.\right. \\
& \left.+\int_{0}^{\infty} \xi \frac{\left[\sinh \xi x e^{-\left(H-x_{i}\right) \xi}-\sinh [(H-x) \xi] e^{\left.-\xi x_{i}\right]}\right.}{\sinh \xi H} J_{0}(\xi r) d \xi\right] \\
& +d_{i}^{x}(t)\left[\frac{1}{R_{i}^{3}}-\frac{3\left(x-x_{i}\right)^{2}}{R_{i}^{5}}\right. \\
& \left.\left.+\int_{0}^{\infty} \xi^{2} \frac{\left.\sinh \xi x e^{-\left(H-x_{i}\right) \xi}+\sinh [(H-x) \xi] e^{-\xi x}\right]}{\sinh \xi H} J_{0}(\xi r) d \xi\right]\right\}
\end{aligned}
$$

The theory proceeds as with all other cases.

Illustrations of the theoretical calculations are shown in Figures 17, 18, 19, and 20.

In Figure 17 the effect of widening the gap between the two infinite plane boundaries is considered. In Figure 17(a), the dimensionless gap is 2.0. In this case the bubble is elongated between the plates during the growth phase, collapsing with an axisymmetric "ring-jet" during the collapse phase. In Figure 17(b) and (c) the plate gaps are 2.5 and 3.0 respectively. Similar phenomena are observed in both the examples, except in these cases the collapsed state of the bubble is much smaller. Chahine [6] observed "ringjet" phenomena in his experiments on cavitation bubbles between two parallel plane boundaries. Although the physical parameters are not available for his experiments, the observed bubble shapes seem to indicate that the bubbles have a much greater "attachment" to the boundary than our theory indicates. It would seem that modelling the growth and collapse of cavitation bubble by a source-dipole approach located at the centroid may not yield realistic bubble shapes if the boundaries are too close. One 

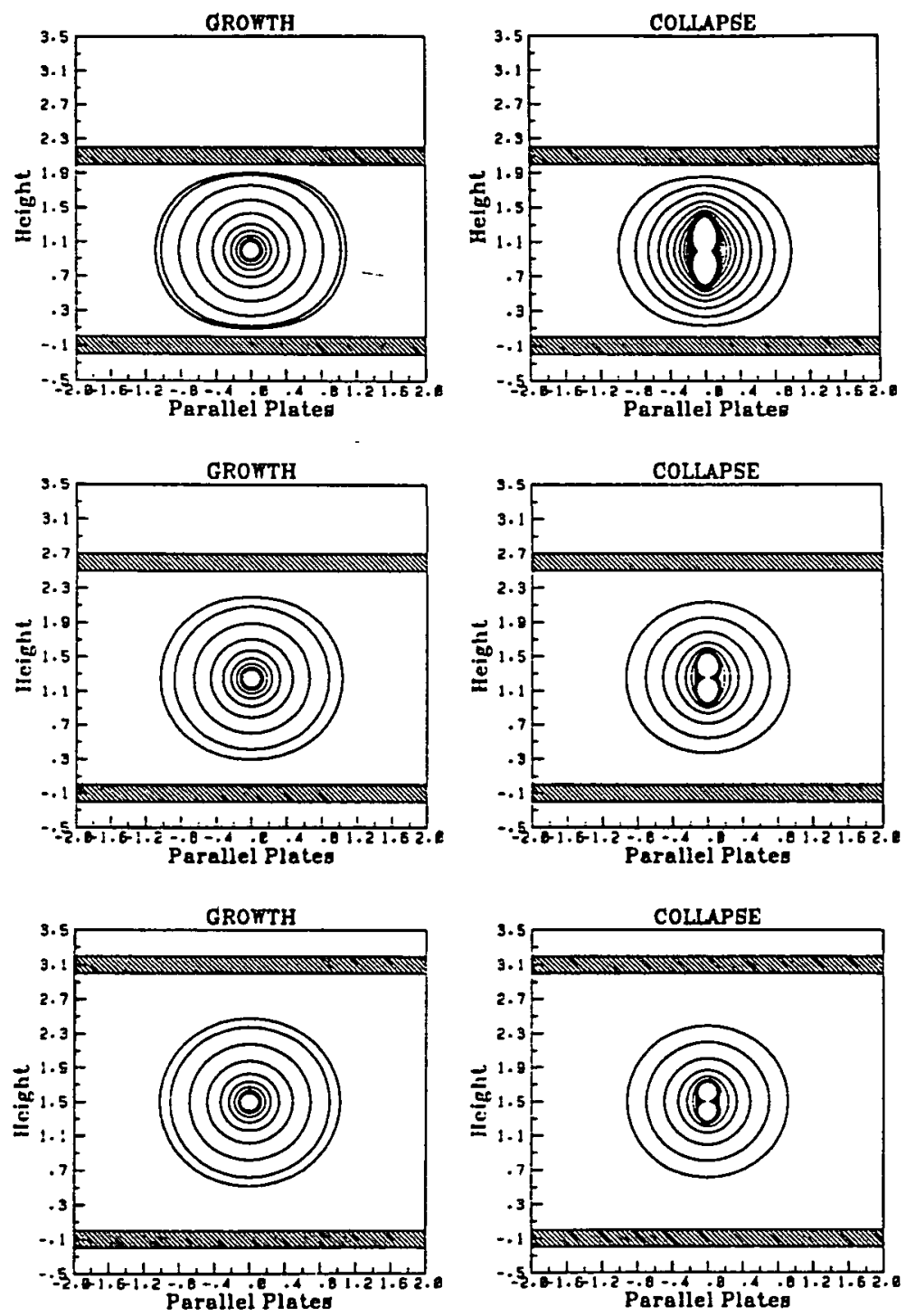

Figure 17 Symmetric Growth and collapse of a single bubble between 2 parallel flat plates. In all cases a ring jet is developed during collapse.

should therefore regard this method as yielding only qualitative information on the bubble characteristics.

In Figure 18.1(a), (b), (c) the growth and collapse of an off-centre bubble is con- 

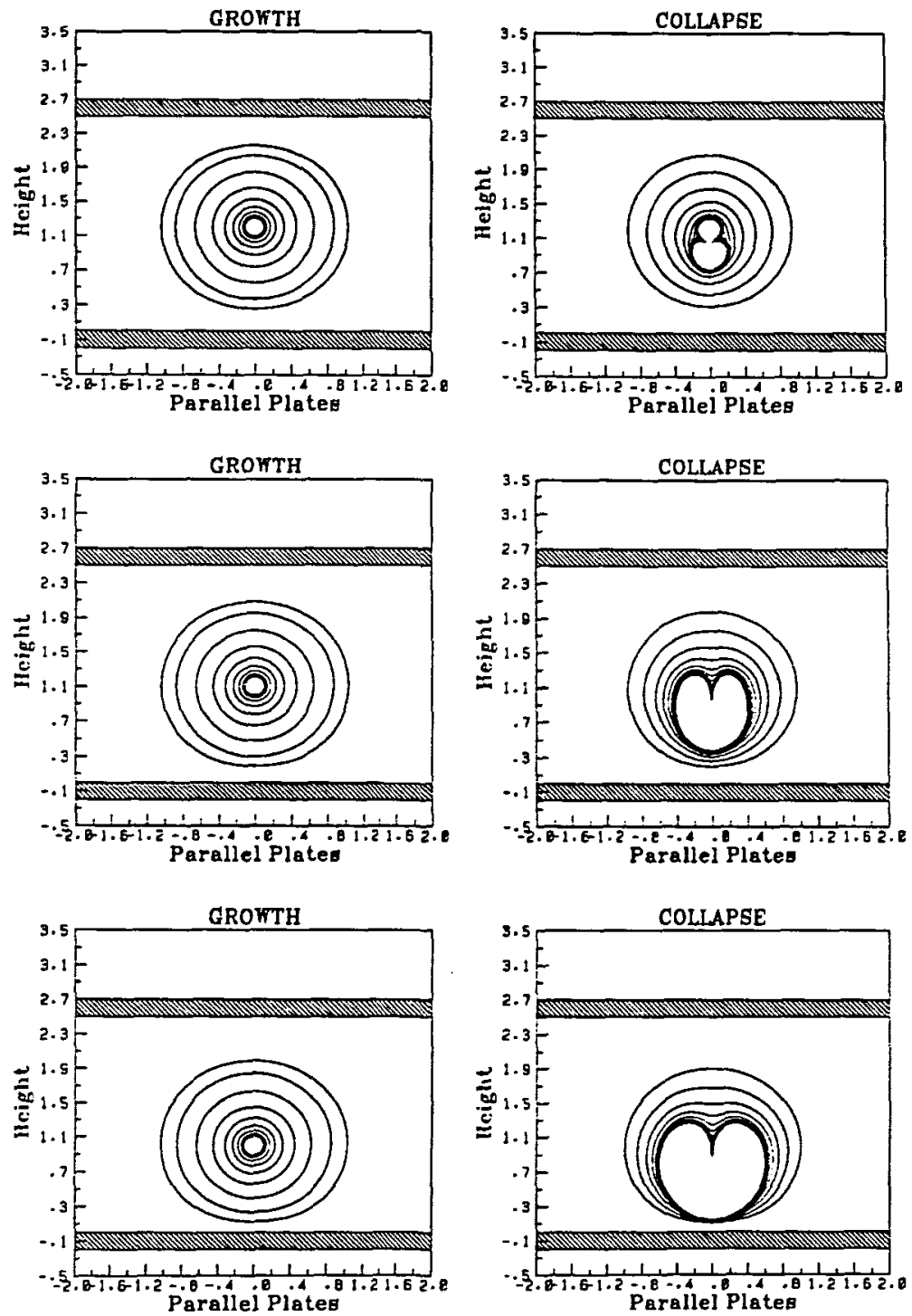

Figure 18.1 Asymmetric growth and collapse between two parallel flat plates. In all cases the bubble is located closer to the lower boundary.

sidered. In Figure 18.1(a), the "ring-jet" collapse is calculated, noting the rather weak Kelvin impulse calculation (see Figure 18.2(a)). In Figures 18.1(b) and 18.1(c), the liquid jet is strongly attracted towards the closest rigid boundary. This is borne out by 

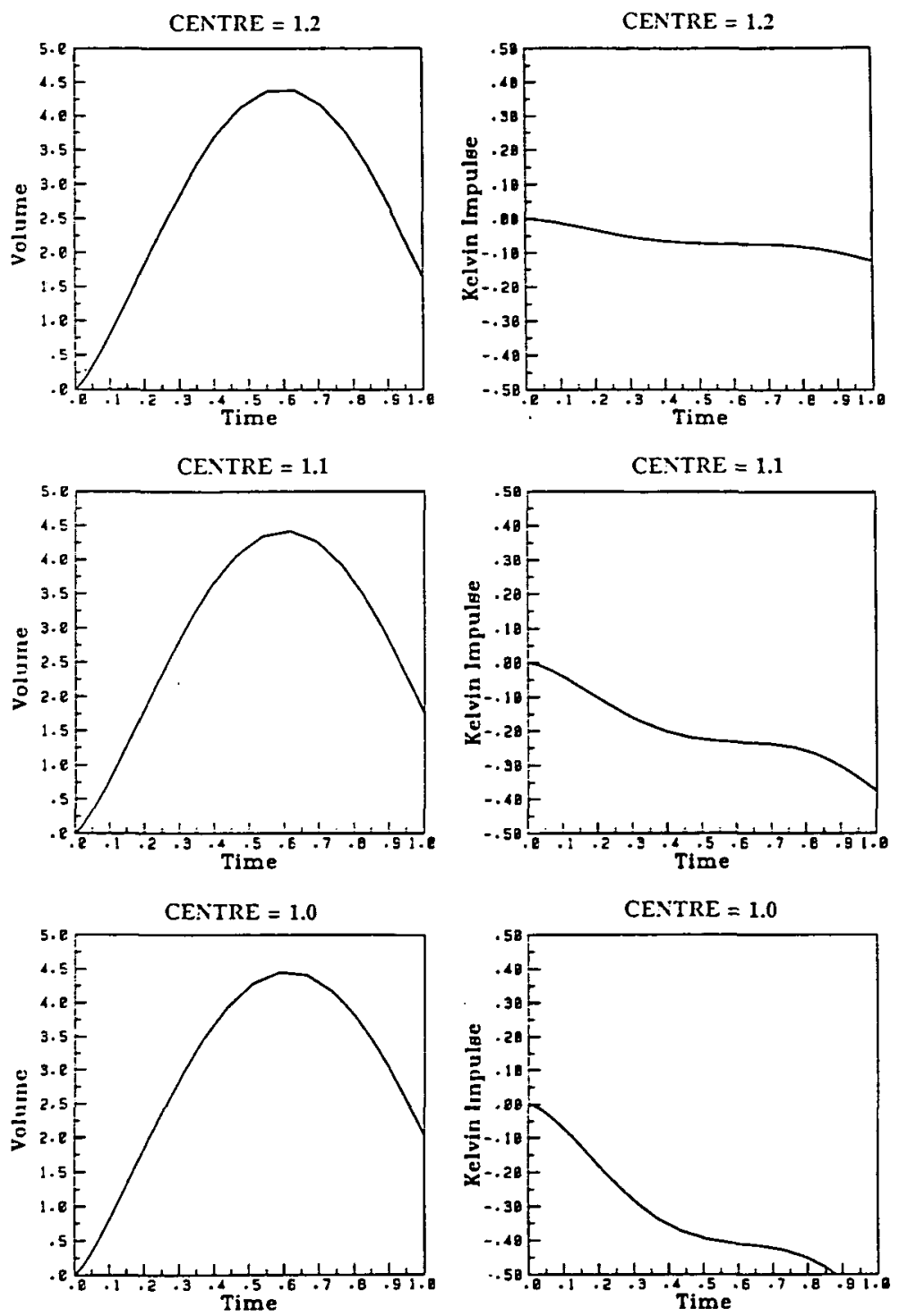

Figure 18.2 Continuous time comparisons of the bubble volumes and their Kelvin Impulses for the bubbles in Figures 18.1(a), 18.1(b) and 18.1(c) respectively.

the much stronger Kelvin impulse values as shown in 18.2(b) and 18.2(c).

Figure 19(a), (b), (c) considers two bubbles between the boundaries. In Figure 19(a) the strong mutual attraction of both the rigid boundary and the 'other' bubbles 
leads to "ring-jet" formation in both bubbles. In Figures 19(b) and (c) the interaction with the adjacent bubble dominates over the boundary effects leading to the jet being directed towards the other bubble.
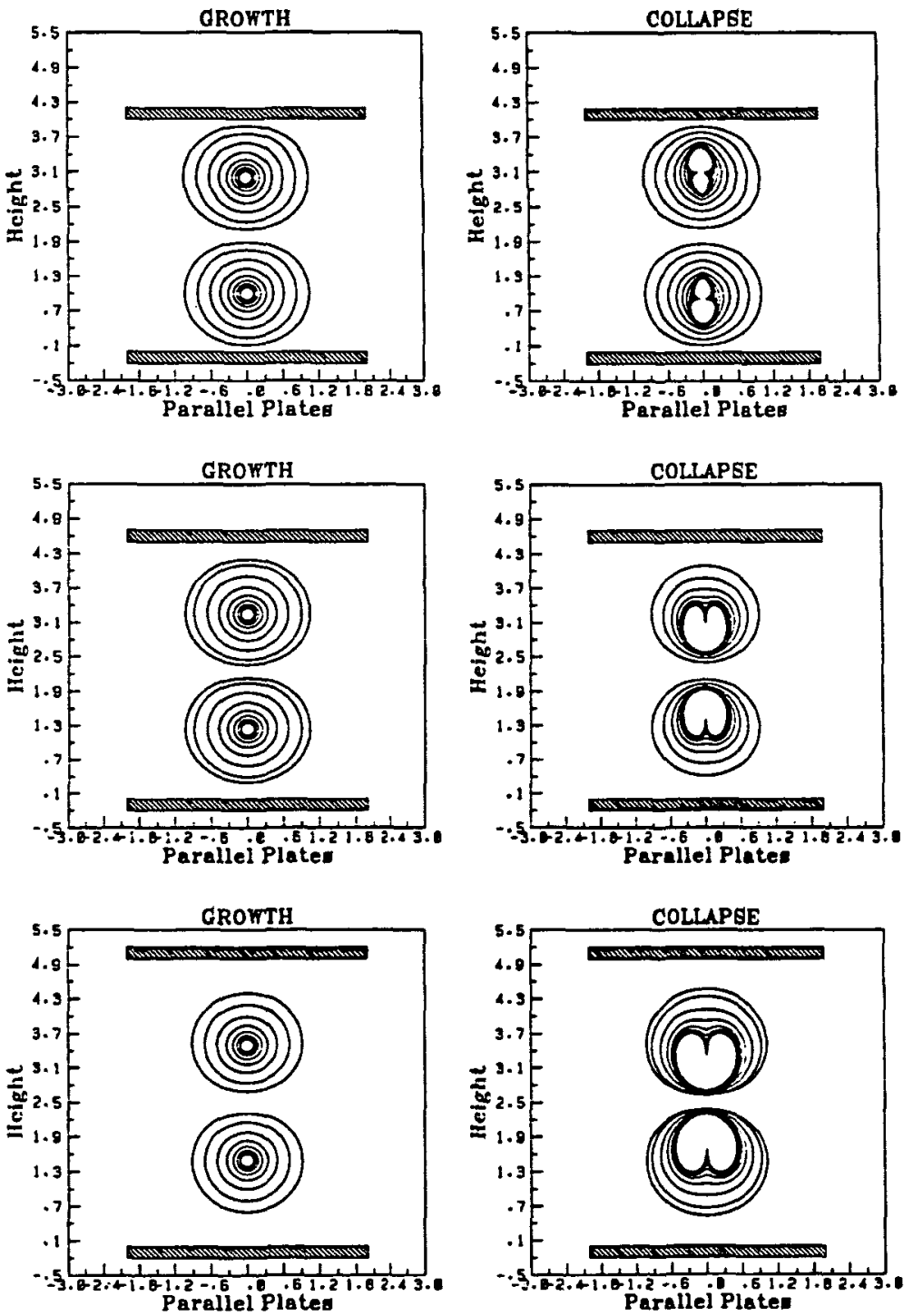

Figure 19 Growth and collapse of 2 symmetrically located bubbles between parallel plates.

In Figure 20 a 3-D graphical illustration of the growth and collapse of four bubbles, 
initially at $\gamma=1,4,7$, and 9 , with bounding parallel plates at 0 and 11 is shown. The bubbles are attracted to the boundary and each other. The bubble at $\gamma=4$, being somewhat more separated proceeds to collapse in a more or less spherical shape, whereas the bubble at $\gamma=9$ maintains much of its maximum volume due to the influence of the nearby bubble $\gamma=7$ and the rigid boundary at $\gamma=11$.

\section{Conclusions}

In this paper a simple method based on a source-dipole approximation is used to model the growth and collapse of cavitation bubbles near rigid boundaries of different geometries. It is found that the method gives a remarkably accurate approximation to the growth and early collapse stages of the lifetime of a bubble. The method becomes inaccurate and inappropriate once the jet has formed. However it does appear to be an economical method for extracting the early growth and collapse characteristics for bubbles close to rigid boundaries. 

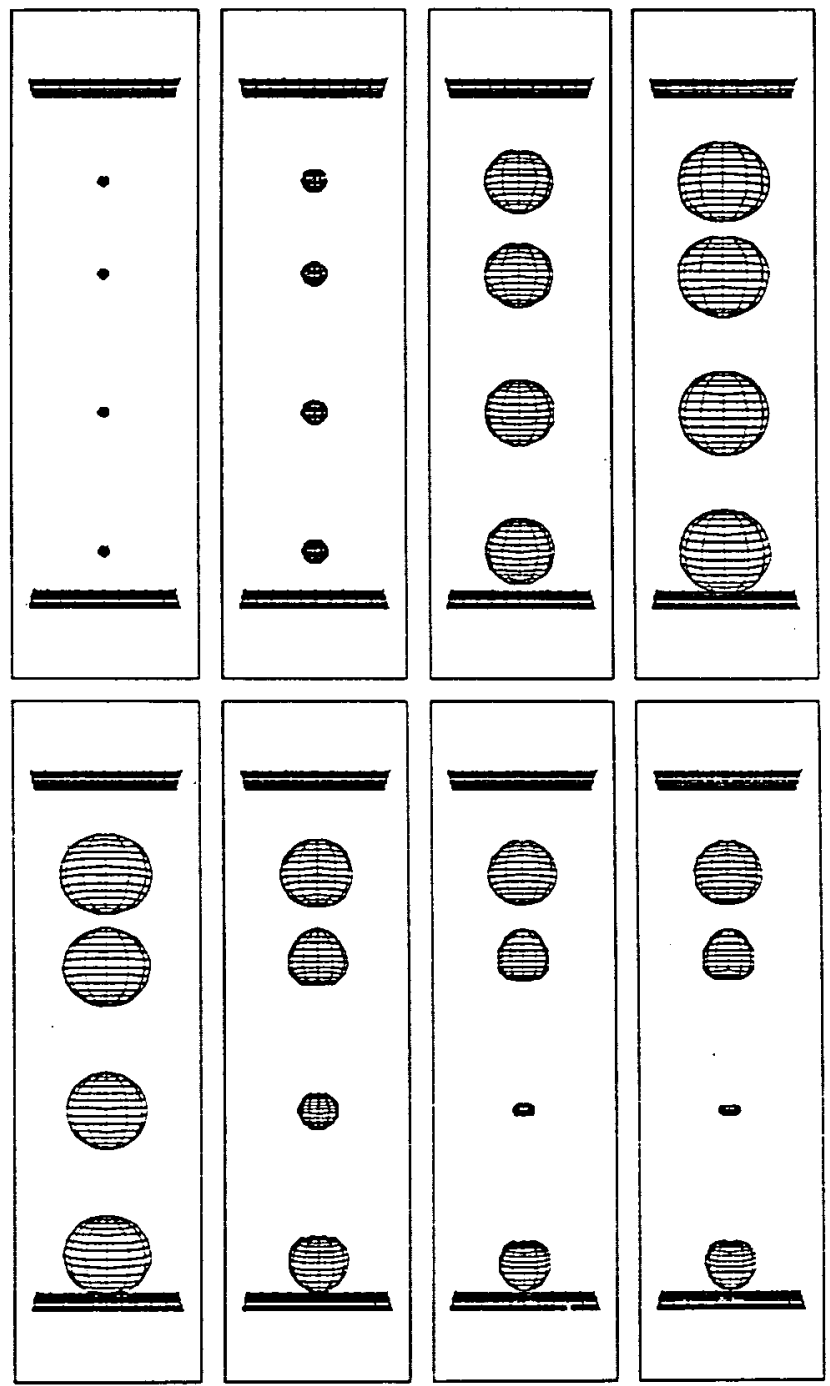

Figure 20 Three dimensional illustrations of the growth and collapse of four bubbles initially located at $\gamma=1,4,7$ and 9 with bounding parallel plates at 0 and 11.

\section{REFERENCES}

[1] M. Abramowitz and I.A. Stegun, Handbook of Mathematical Functions (Dover, New York, 1965). 
[2] J.R. Blake, 'The Kelvin impulse: applications to cavitation bubble dynamics', J. Austral. Math. Soc. Ser. B 30 (1988).

[3] J.R. Blake and D.C. Gibson, 'Growth and collapse of a vapour cavity near a free surface', J. Fluid Mech. 111 (1981), 123-140.

[4] J.R. Blake and D.C. Gibson, 'Cavitation bubbles near boundaries"', Ann. Rev. Fluid Mech. 19 (1987), 99-123.

[5] J.R. Blake, B.B. Taib and G. Doherty, 'Transient cavities ner boundaries, Part 1. Rigid Boundary', J. Fluid Mech. 170 (1986), 479-497.

[6] G.L. Chahine, 'Experimental and asymptotic study of non-spherical bubble collapse', Appl. Sci. Res. 38 (1982), 187-197.

[7] G.L. Chahine and Y.T. Shen, 'Bubble dynamics and cavitation inception in cavitation susceptibility meters', Trans ASME 108 (1986), 444-452.

[8] L. Euler, 'Theorie plus complette des machines qui sont mises en mouvement par la reaction de l'eau' 10, pp. 227-295.

[9] D.C. Gibson and J.R. Blake, 'The growth and collapse of bubbles near deformable surfaces', Appl. Sci. Res. 38 (1982), 215-224.

[10] L. Landweber, 'Axisymmetric potential flow in a circular tube', J. Hydronautics 8 (1974), 137-145.

[11] J. Lighthill, An Informal Introduction to Theoretical Fluid Mechanics (Oxford University Press, Oxford, 1986).

[12] M.S. Plesset, 'The dynamics of cavitation bubbles', J. Appl. Mech 16 (1949), 277-282.

[13] M.S. Plesset and R.B. Chapman, 'Collapse of an initially spherical vapour cavity in the neighbourhood of a solid boundary', J. Fluid Mech. 47 (1971), 283-290.

[14] A. Prosperetti, 'Bubble dynamics: a review and some recent results', Appl. Sci. Res. 38 (1982), 145-164.

[15] A. Prosperetti, 'On the dynamics of non-spherical bubbles', in Cavitation and inhomogeneities in underwater acoustics, Editor W. Lauterborn (Springer, Berlin, Heidelberg, New York, 1980).

[16] Lord Rayleigh, 'On the pressure developed in a liquid during the collapse of a spherical void', Phil. Mag. 34 (1917), 94-98.

[17] O. Reynolds, 'Experiments showing the boiling of water in an open tube at ordinary temperatures', Brit. Assoc. Adv. Sci. Rep. 564 (1894).

[18] A. Shima and K. Nakajima, 'The collapse of a non-hemispherical bubble attached to a solid wall', J. Fluid Mech. 80 (1978), 369-391.

[19] A. Shima, Y. Tomita, D.C. Gibson and J.R. Blake, 'The growth and collapse of cavitation bubbles near composite surfaces', J. Fluid Mech. 203 (1989), 199-214.

[20] A.Sommerfeld, 'Mathematische Theorie der Diffraction', Mathematische Annalen 47 (1896), 317-374.

[21] Y. Tomita and A. Shima, 'Mechanisms of impulsive pressure generation and damage pit formation by bubble collapse', J. Fluid Mech. 169 (1986), 535-564. 
Department of Mathematics La Trobe University Bundoora Vic 3083

Australia
School of Mathematics and Statistics

University of Birmingham

Edgbaston B15 2TT

United Kingdom 